\title{
On the Fidelity of Large-Eddy Simulation of Shallow Precipitating Cumulus Convection
}

\author{
Georgios Matheou AND DANiel CHUnG \\ Jet Propulsion Laboratory, California Institute of Technology, Pasadena, California \\ LOUISE NUIJENS AND BJORN STEVENS \\ Max Planck Institute for Meteorology, Hamburg, Germany, and Department of Atmospheric and Oceanic Sciences, \\ University of California, Los Angeles, Los Angeles, California \\ JOAO TEIXEIRA \\ Jet Propulsion Laboratory, California Institute of Technology, Pasadena, California
}

(Manuscript received 19 August 2010, in final form 3 February 2011)

\begin{abstract}
The present study considers the impact of various choices pertaining to the numerical solution of the governing equations on large-eddy simulation (LES) prediction and the association of these choices with flow physics. These include the effect of dissipative versus nondissipative advection discretizations, different implementations of the constant-coefficient Smagorinsky subgrid-scale model, and grid resolution. Simulations corresponding to the trade wind precipitating shallow cumulus composite case of the Rain in Cumulus over the Ocean (RICO) field experiment were carried out. Global boundary layer quantities such as cloud cover, liquid water path, surface precipitation rate, power spectra, and the overall convection structure were used to compare the effects of different discretization implementations. The different discretization implementations were found to exert a significant impact on the LES prediction even for the cases where the process of precipitation was not included. Increasing numerical dissipation decreases cloud cover and surface precipitation rates. For nonprecipitating cases, grid convergence is achieved for grid spacings of $20 \mathrm{~m}$. Cloud cover was found to be particularly sensitive, exhibiting variations between different resolution runs even when the mean liquid water profile had converged.
\end{abstract}

\section{Introduction}

Shallow cumulus convection is frequent over the World Ocean, and as one of the principal elements of the Hadley cell it plays an important role in the global circulation (e.g., Tiedtke 1989; Siebesma 1998; Stevens 2005). Convection in the cumulus-topped boundary layer is characterized by the relatively small scales of the individual cumulus that are mediated by the overall convection structure and in particular by updrafts emanating near the surface $(1-3 \mathrm{~km})$. Although the fundamental dynamics had been identified early (Bjerknes 1938; Stommel 1947; Malkus 1954), an accurate statistical description of the boundary layer ensemble

Corresponding author address: Georgios Matheou, Jet Propulsion Laboratory, 4800 Oak Grove Dr., Pasadena, CA 91109. E-mail: georgios.matheou@jpl.nasa.gov remains elusive (e.g., Randall et al. 2003; Arakawa 2004; Bony et al. 2004; Teixeira et al. 2008). Yet, such coarsegrained descriptions are necessary to represent, or parameterize, shallow convection in weather forecast and climate models.

A large part of the difficulty in formulating a universal parameterization is a result of the complex interactions among processes spanning a wide spectrum of scales. Large-scale atmospheric processes (such as winds, subsidence, and surface conditions) bring about an environment where cloud-scale motions dominate vertical transport. In turn, vertical transport depends on the smallscale processes of entrainment and mixing.

High-resolution simulation is an essential element in boundary layer studies and is designed to explore the interplay between the small-scale turbulent dynamics associated with clouds and thermals and the larger-scale environmental conditions that give rise to them. Largeeddy simulation (LES) models have been widely used in 
fundamental investigations, such as those of Sommeria (1976), Cuijpers and Duynkerke (1993), Siebesma and Jonker (2000), and Heus and Jonker (2008), and in development and evaluation of parameterizations of the cumulus-topped boundary layer (e.g., Siebesma and Holtslag 1996; Siebesma et al. 2003; Soares et al. 2004; Neggers et al. 2009). LES is a turbulence modeling technique in which most of the energy-containing motions are explicitly computed while motions smaller than a certain cutoff scale, usually taken equal to the computational grid spacing, are modeled. In LES a significant range of scales can be computed, presently $L / \Delta x=\mathcal{O}\left(10^{3}\right)$ for state-of-the-art LES, where $L$ is the horizontal extent of the computational domain and $\Delta x$ is the grid spacing, allowing for the important effects of horizontal variability to be explicitly simulated. These characteristics make LES particularly attractive in numerical studies of cumulus convection.

Although the accuracy of LES predictions for dry and nonprecipitating cumulus-topped boundary layers has been established (e.g., Schmidt and Schumann 1989; Nieuwstadt et al. 1993; Siebesma et al. 2003), the performance of LES for the case of precipitating cumulus convection remains largely undocumented. Precipitation in maritime cumulus is common (e.g., Rauber et al. 1996; Short and Nakamura 2000; Nuijens et al. 2009) and can have a significant impact on the development of the boundary layer as it furnishes a path for the vertical transport-albeit downward-of water mass and energy in addition to turbulent transport. From a modeling perspective, precipitation can be viewed as a process exacerbating the nonlinearity of turbulent transport through a strong two-way coupling between the two processes. Therefore, it is expected that simulation of precipitating shallow convection will exhibit larger sensitivities to modeling choices compared to nonprecipitating cases. Understanding these aspects and their connection to the flow physics is a necessary first step toward predictive simulations that will allow for an in-depth investigation of the dynamics of precipitating cumulus.

The greater part of previous LES investigations of cumulus convection involved nonprecipitating cases. Brown (1999) and Stevens et al. (2002) focused on the effects of grid resolution and computational domain size and found that grid resolution resulted in improved representation of the dynamics of individual cloud evolution while larger domains allowed large-scale organization, which in turn affected ensemble boundary layer properties. These studies, in accord with the results of the Barbados Oceanographic and Meteorological Experiment (BOMEX) model intercomparison study (Siebesma et al. 2003), concluded that domainmean variables exhibited less variation with respect to simulation parameters than quantities more representative of the convection organization, such as cloud cover. Numerical dissipation was also recognized to play a role in these types of simulations. However, numerical dissipation is essentially an implicit subgrid-scale (SGS) model because of its property to dissipate kinetic energy and the variance of scalars, and its effects are difficult to untangle from the explicit model and thus to assess independently (e.g., Siebesma et al. 2003; Piotrowski et al. 2009).

The present LES runs correspond to the composite case of the Rain in Cumulus over the Ocean (RICO) field experiment (Rauber et al. 2007) that was part of the Global Energy and Water Cycle Experiment (GEWEX) Cloud System Study (GCSS) model intercomparison study (VanZanten et al. 2011). The main aim of this study is to investigate the dependence of the predicted state of the boundary layer on common discretization choices and make the connection between the observed differences and boundary layer physics. This is accomplished by using the University of California, Los Angeles (UCLA)-LES code (Stevens et al. 1999, 2005; Stevens and Seifert 2008), one of the models that took part in the RICO model intercomparison study. Moreover, the goal of the present study is not to optimize, or "tune," the model configuration, but rather to investigate the outcomes of common model configurations with the expectation that the results will be broadly relevant in LES of atmospheric boundary layers. The discretization choices concern the momentum and scalar advection terms (i.e., dissipative versus nondissipative schemes), discretization of the subgrid-scale tensor, and grid resolution. For the purposes of the comparison only general metrics will be used, such as time evolution of cloud cover, liquid water path (LWP), and surface precipitation rate, and cloud organization and power spectra at the end of the simulation. Simulations will be mostly compared to each other with limited references to observations or other model results as this will be the task of the RICO model intercomparison study and future extensions of this work.

Precipitating LES cases that are more closely connected to the present discussion are those of Stevens and Seifert (2008) and Xue et al. (2008) and the one by Abel and Shipway (2007) that used a coarser-grained (compared to LES) cloud-resolving model. The simulations of Abel and Shipway (2007) and Stevens and Seifert (2008) correspond to conditions taken from the RICO field study, while Xue et al. (2008) based their setup on the Atlantic Tropospheric Experiment (ATEX) intercomparison case (Stevens et al. 2001). Whereas Stevens and Seifert (2008) and Xue et al. (2008) focused on the effects of microphysics, the present study concerns 
the characteristics of turbulent transport modeling in shallow precipitating cumulus convection.

\section{Model description}

The LES computer code used in the present study is based on the UCLA-LES code (Stevens et al. 1999, 2005; Stevens and Seifert 2008). However, several important differences exist between the current and previous variants of the implementation.

In the present model the anelastic approximation (Ogura and Phillips 1962; Vallis 2006) of the Favrefiltered (density-weighted) Navier-Stokes equations is used. The subgrid-scale stress tensor and scalar fluxes are modeled using an eddy diffusivity assumption. The eddy diffusivity for all scalar variables is related to the SGS momentum diffusivity $\nu_{t}$ through the constant turbulent Prandtl number $\operatorname{Pr}_{t}=1 / 3$.

The turbulence closure originally introduced by Smagorinsky (1963) with Lilly (1962) buoyancy correction is used to compute the turbulent diffusivity. The Smagorinsky coefficient is assumed constant and equal to $C_{S}=0.23$, as in several previous applications of the UCLA-LES model. Although Lilly's (1962) modification takes into account the subgrid buoyancy to correct the turbulent diffusivity, it still neglects the anisotropy of the subgrid-scale mixing that results in stratified conditions. This approximation is expected to be adequate in highresolution simulations of cumulus convection because the boundary layer is only moderately stably stratified.

Near the surface, the underlying assumptions of LES modeling weaken as the characteristic length scale of the most energetic motions becomes comparable to, or even smaller than, the grid spacing. The constant-coefficient Smagorinsky closure has been shown to excessively dissipate turbulent motions in this region (e.g., Mason 1994; Cabot and Moin 1999); therefore, a damping function is used to reduce the magnitude of the turbulent diffusivity by modifying the characteristic resolved-scale length scale following Mason and Thomson (1992). Moreover, surface turbulent fluxes are parameterized and a slip/nopenetration condition is used for the resolved transport because the grid spacing does not decrease to resolve the no-slip boundary condition at the surface.

To compute the latent heat exchange in the two-phase fluid, condensation is assumed to occur when the mean state in each grid cell becomes saturated, a fact that implies a uniform subgrid distribution (all or nothing) of humidity and temperature.

The process of warm-rain precipitation is parameterized using the two-moment bulk microphysical scheme of Seifert and Beheng (2001). Although the accuracy of the microphysical scheme that is used to parameterize the evolution of rain drops is not critical here, the process of precipitation is important in the development of the boundary layer.

\section{Discrete model}

In large-eddy simulation, numerical solutions are sought by replacing the continuous operators of the governing equations with discrete analogs. The resulting LES solutions are distinctive in the sense that the solution is always underresolved, meaning that flow fields always exhibit significant fluctuations at scales close to the grid resolution. Accordingly, increasing the grid resolution does not make the LES solution "smoother" and any advantages gained are because of improvements in the performance of the SGS model (e.g., Pope 2004; Matheou et al. 2010). As a consequence, when finite differences are used to discretize the differential operators, the formal order of accuracy of the approximation is inconsequential and what is relevant in LES is the dispersion characteristics, as shown in the seminal work of Ghosal $(1996,1999)$.

In the present study, of primary concern are the discretization choices of the nonlinear terms, that is, advection and SGS tensor/fluxes, in the transport equations and the effect they exert on the prediction of the flow. By controlling for two important parameters-(a) the numerical dissipation of the advection term and (b) the discretization of the SGS model-a deeper insight into the performance of the LES is accomplished.

In general, discretization of the spatial derivatives in this model is done using second-order finite differences on an Arakawa C-grid and time integration is accomplished using the low-storage third-order Runge-Kutta method of A. Wray in Spalart et al. (1991). The time step is adjusted such as to maintain a constant CourantFriedrichs-Lewy (CFL) number of 0.3 .

\section{1) AdVECTION TERMS}

In this model advection terms are always discretized using second-order centered differences (Harlow and Welch 1965). Two types of advection schemes are explored for the total water and liquid water potential temperature equation: second-order centered differences and a monotone advection scheme.

The second-order centered differences scheme is implemented in flux form and has the advantage of being nondissipative and energy conserving. That is, the discrete kinetic energy and variance for scalars are conserved (Piacsek and Williams 1970; Morinishi et al. 1998). In simulations of turbulent flows the scheme's conservation properties are essential. The main disadvantage of 
the nondissipative scheme is that it can produce unphysical dispersive spatial oscillations.

On the other hand, the monotone flux-limited advection scheme (Toro 1999, p. 470) suppresses the development of unphysical oscillations by artificially introducing a diffusive contribution to the advection operator. Because the transport equations are nonhomogeneous, as a result of the condensation/evaporation source terms, strict monotonicity is not preserved as discussed in Grabowski and Smolarkiewicz (1990). The introduction of artificial dissipation can have an adverse effect on the evolution of the turbulent flow because of the excessive and unphysical destruction of scalar variance and kinetic energy even in simulations of relatively simple flows using high-order low-dissipation schemes (e.g., Pantano et al. 2007). In several previous investigations with the UCLA-LES code the monotone advection for scalar variables was utilized (e.g., Siebesma et al. 2003; Stevens et al. 2005; Savic-Jovcic and Stevens 2008; Stevens and Seifert 2008).

It is important to note that even when total water and liquid water potential temperature are advected using the centered scheme, rain mass and rain number are always advected using the monotone scheme. This choice is necessary in this model because negative rain mass resulting from the spurious oscillations is difficult to handle and thus negative values need to be "clipped" to zero. However, clipping the undershoots causes a break in the conservation of water as the overshoots cannot be detected and are not clipped (e.g., Skamarock and Weisman 2009).

In LES of shallow cumulus convection where very sharp gradients are scattered and short-lived (e.g., at the edges of clouds penetrating the inversion) the nondissipative advection scheme is expected to perform well and dispersive errors are outweighed by the energy conservation properties.

\section{2) SGS TERMS}

Although far less consideration was given to discretization implementations for the SGS than the advection terms, the SGS model implementation can be important in LES. This is not only for numerical stability considerations (e.g., Kamakoti and Pantano 2009); in addition, as Viré and Knaepen (2009) point out, the performance of the SGS closure can change significantly depending on the discretization adopted. Viré and Knaepen (2009) refer to discretization "errors" that result from different spatial derivative approximations. These errors are quantified with respect to a reference LES solution of decaying homogeneous isotropic turbulent flow computed using a spectral method. However, in the present discussion the term "error" will not
TABLE 1. Summary of the cases simulated. The labeling of the cases takes the form [SGS model implementation]-[advection scheme]- $\Delta x$-microphysics, where the advection scheme tag is $\mathrm{C}$ for centered or $\mathrm{M}$ for monotone and when the process of precipitation is included the microphysics tag is $\mathrm{M}$ (otherwise it is X). A single run was conducted without the Galilean transform and is denoted by $-\mathrm{NG}$.

\begin{tabular}{|c|c|c|c|c|c|}
\hline Case & Advection & $\begin{array}{c}\text { SGS } \\
\text { model }\end{array}$ & Microphysics & $\begin{array}{c}L \\
(\mathrm{~km})\end{array}$ & $\begin{array}{l}\Delta x \\
(\mathrm{~m})\end{array}$ \\
\hline SMC-C-80-M & Centered & $\mathrm{SMC}$ & On & 20.48 & 80 \\
\hline SMC-C-40-M & Centered & SMC & On & 20.48 & 40 \\
\hline SMC-C-20-M & Centered & SMC & On & 20.48 & 20 \\
\hline SMC-C-80-X & Centered & $\mathrm{SMC}$ & Off & 20.48 & 80 \\
\hline SMC-C-40-X & Centered & SMC & Off & 20.48 & 40 \\
\hline SMC-C-20-X & Centered & SMC & Off & 20.48 & 20 \\
\hline SMC-C-10-X & Centered & SMC & Off & 20.48 & 10 \\
\hline SMC-M-40-M & Monotone & SMC & On & 20.48 & 40 \\
\hline SMC-M-40-X & Monotone & SMC & Off & 20.48 & 40 \\
\hline SMC-M-40-X-NG & Monotone & SMC & On & 20.48 & 40 \\
\hline SMV-C-40-M & Centered & SMV & On & 20.48 & 40 \\
\hline SMV-C-40-X & Centered & SMV & Off & 20.48 & 40 \\
\hline SMV-M-40-M & Monotone & SMV & On & 20.48 & 40 \\
\hline SMV-M-40-X & Monotone & SMV & Off & 20.48 & 40 \\
\hline
\end{tabular}

be adopted because a reference solution is not available and we are concerned only with finite difference approximations.

Two implementations of the constant-coefficient Smagorinsky SGS model are used. They differ in the way the turbulent diffusivity $\nu_{t}$ is computed and the place it is defined on the grid. The first implementation defines $\nu_{t}$ at cell centers, the same location as all scalar variables. The elements of the rate of strain tensor are evaluated also at cell centers using centered differences. The diffusivities are then interpolated at the locations where SGS stresses are calculated. This implementation of the SGS model will be referred to as Smagorinsky centered (SMC).

The second implementation, named Smagorinsky vertical (SMV), is more adjusted to flows with strong vertical gradients, such as those encountered near solid boundaries or in very stably stratified regions. The implementation follows that of Mason and Callen (1986) where the diffusivity is defined at the same location as the vertical velocity. The components of the rate of strain tensor are evaluated at the midpoints between the corresponding velocity components and then squared, and the squares are finally interpolated onto the $w$ points.

Implementation SMV was used in previous studies with the UCLA-LES code. The new implementation, SMC, has the advantage of being more "isotropic," as the turbulent viscosity is defined at cell centers and the same interpolation scheme is used to evaluate SGS fluxes at all cell faces and vertices. Moreover, because 

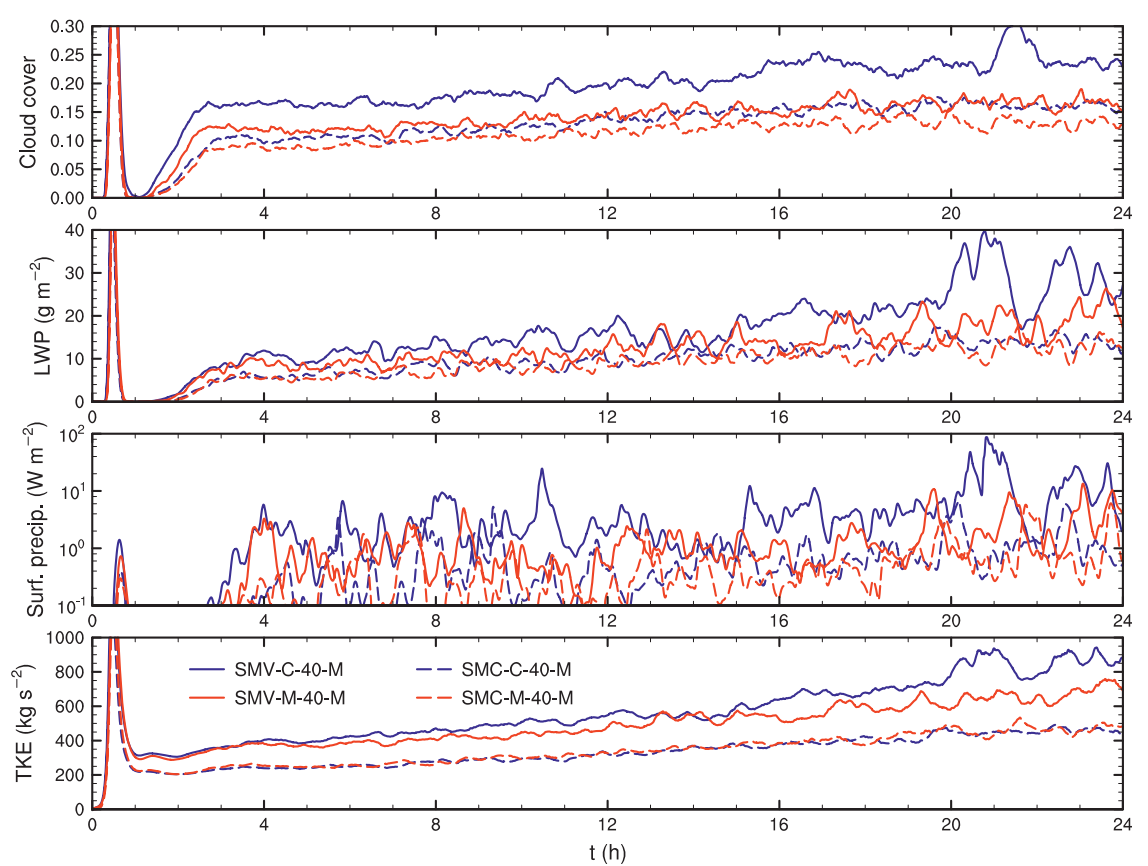

FIG. 1. Time evolution of cloud cover, LWP, surface precipitation rate, and vertically integrated TKE for runs with microphysics and $\Delta x=40 \mathrm{~m}$, for nondissipative advection (blue) and the dissipative monotone scheme (red), for SGS model implementation SMV (solid) and implementation SMC (dashed).

in implementation SMV $\nu_{t}$ resides at the boundary between the grid cells, it results in some ambiguity with regards to the thermodynamic state of the fluid at that point. For example, air is assumed to be saturated when computing $\nu_{t}$ if both neighboring cells are saturated.

The details of the discretization and the variable arrangement on the computational grid are documented in further detail in appendix A.

\section{Simulations}

The simulations correspond to the composite conditions of the RICO field experiment (VanZanten et al. 2011). For all cases, the computational domain has dimensions of 20.48 $\times 20.48 \times 4 \mathrm{~km}^{3}$ with periodic lateral boundary conditions. The grid spacing is uniform in all three directions (grid aspect ratio is always unity). Two sets of simulations were carried out, one with microphysics and one without, in order to separate the effects of precipitation on the evolution of the boundary layer. All simulations were run for $24 \mathrm{~h}$. The largest simulation encompasses $1024 \mathrm{CPU}$ cores and requires roughly 1.5 million CPU hours to complete.

Following the setup of the GCSS case, a Galilean translation velocity of -6 and $-4 \mathrm{~m} \mathrm{~s}^{-1}$ in the zonal and meridional directions, respectively, is added in almost all runs in order to reduce the numerical dissipation of the monotone advection scheme. Instead of including a discontinuous initial random perturbation near the surface, as is commonly used in this type of simulations, a continuous isotropic random perturbation is introduced as described in appendix B. The perturbation with the smallest length scale, perturbation $\mathrm{A}$, was used in all runs.

Table 1 summarizes the cases simulated. The naming of the cases takes the form [SGS model implementation][advection scheme]- $\Delta x$-[microphysics], where the advection-scheme tag is $\mathrm{C}$ for centered or $\mathrm{M}$ for monotone

TABLE 2. Average surface precipitation rate $\left(P_{\text {srf }}\right)$, cloud cover $(\mathrm{CC}), \mathrm{LWP}$, and vertically integrated TKE during the last $4 \mathrm{~h}$ of the simulations, $t=20-24 \mathrm{~h}$.

\begin{tabular}{|c|c|c|c|c|c|c|}
\hline Case & Advection & SGS model & $P_{\text {srf }}\left(\mathrm{W} \mathrm{m}^{-2}\right)$ & $\mathrm{CC}$ & $\operatorname{LWP}\left(\mathrm{g} \mathrm{m}^{-2}\right)$ & TKE $\left(\mathrm{kg} \mathrm{s}^{-2}\right)$ \\
\hline SMC-C-40-M & Centered & SMC & 1.5 & 0.16 & 13.9 & 453 \\
\hline SMC-M-40-M & Monotone & SMC & 1.3 & 0.13 & 12.6 & 464 \\
\hline SMV-C-40-M & Centered & SMV & 16.9 & 0.24 & 29.0 & 857 \\
\hline SMV-M-40-M & Monotone & SMV & 3.2 & 0.17 & 19.0 & 667 \\
\hline
\end{tabular}


(a) SMC-C-40-M

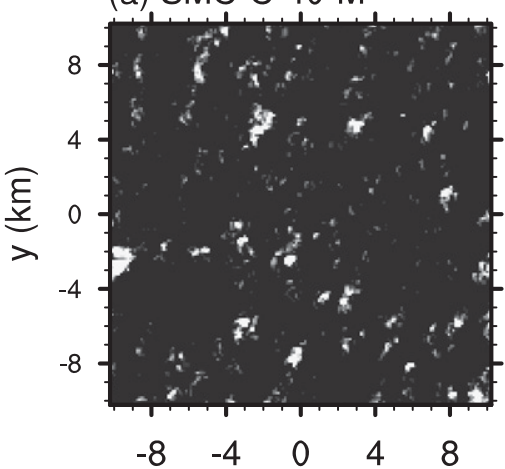

(c) SMC-C-40-X

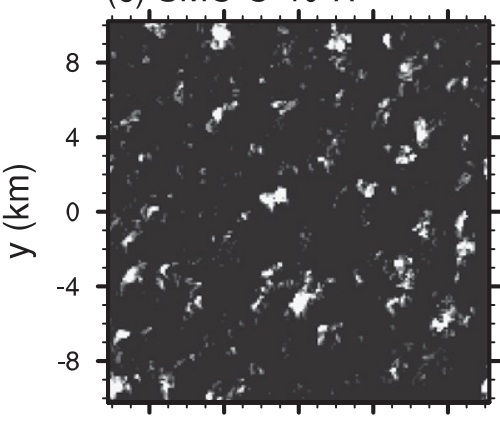

$\begin{array}{lllll}-8 & -4 & 0 & 4 & 8\end{array}$

(e) SMV-C-40-M

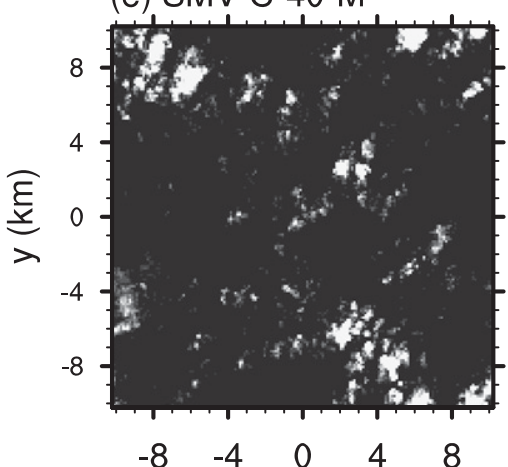

(g) SMV-C-40-X

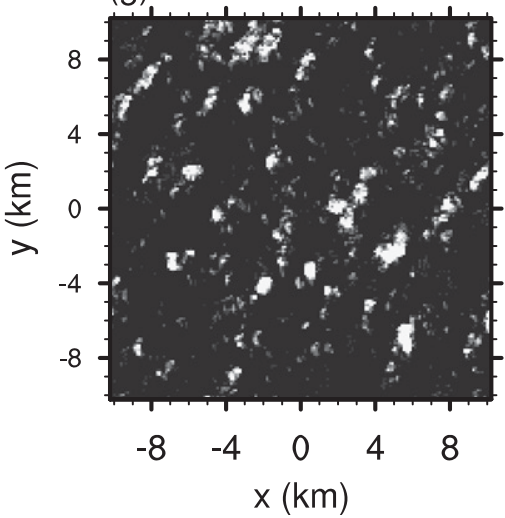

(b) SMC-M-40-M

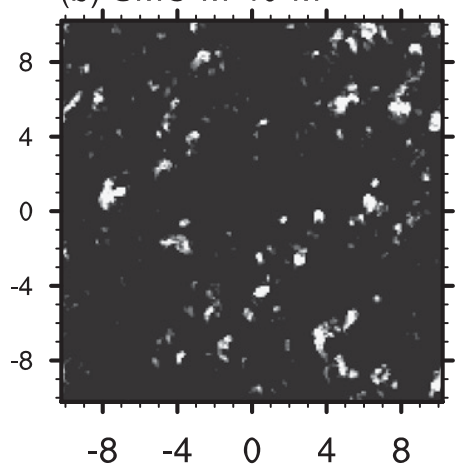

(d) SMC-M-40-X

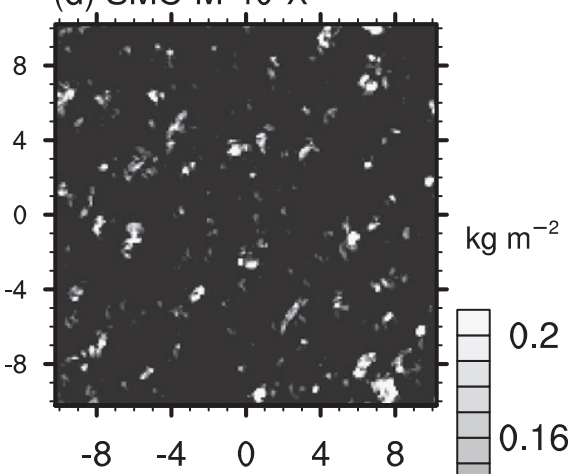

(f) SMV-M-40-M

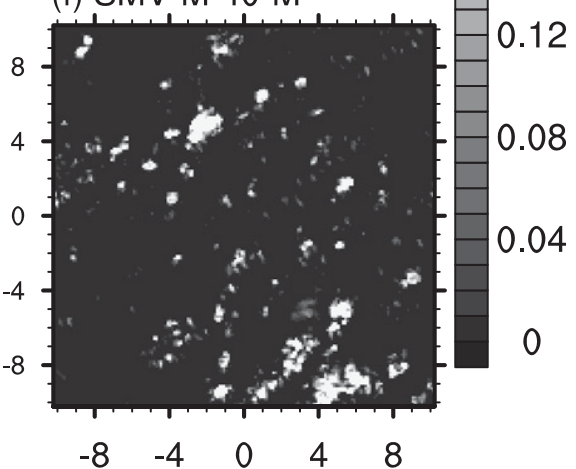

(h) SMV-M-40-X

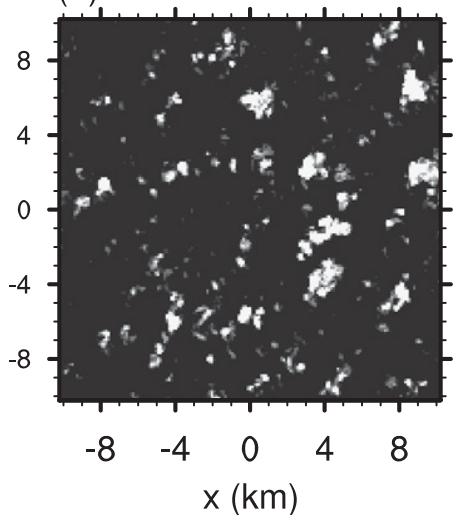

FIG. 2. Cloud LWP at the end of the simulation, $t=24 \mathrm{~h}$. The panels of the left column correspond to LES with the nondissipative advection and show consistently higher cloud cover than those with the monotone scheme. 


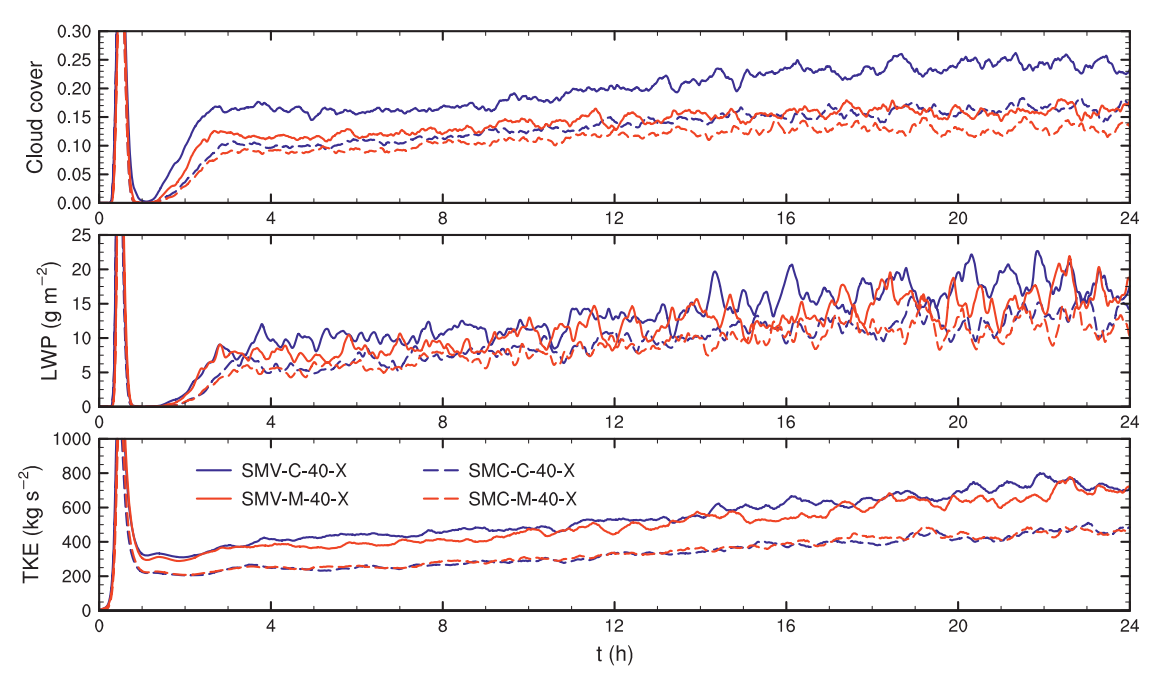

FIG. 3. As in Fig. 1, but for time evolution of cloud cover, LWP, and vertically integrated turbulent kinetic energy for runs without microphysics and $\Delta x=40 \mathrm{~m}$.

and when the process of precipitation is included the microphysics tag is $\mathrm{M}$, and otherwise it is $\mathrm{X}$.

\section{a. Numerical dissipation and subgrid-scale model implementation}

The first set of simulations investigates the effects of numerical dissipation and SGS model implementation while keeping the grid spacing constant, $\Delta x=40 \mathrm{~m}$. To discern the influence of microphysics, runs with and without microphysics are performed. This results in a total of eight simulations.

Results show a significant influence of both numerical dissipation and SGS implementation on the structure of convection. For the present case of shallow cumulus, cloud cover can be a proxy of convection structure. Cloud cover is defined as the fraction of columns that contain at least one cell at any level with cloud water mixing ratio larger than $1 \times 10^{-5}$. LES using the monotone scheme was found to predict consistently lower cloud cover than centered differences as shown in Fig. 1 and Table 2. The sensitivity to numerical dissipation appears to be modulated by the choice of SGS implementation. For implementation SMV cloud cover decreases from about 0.24 to 0.17 , whereas for SMC the change is from about 0.16 to 0.13 . The same trend is observed for liquid water path. These are important differences given the fact that the nominal conditions (second-order finite differences and Smagorinsky SGS model) are the same for all simulations.

The variability of the boundary layer statistics is explored in appendix B using a group of simulations with varying initial conditions. The simulations of appendix B show that the differences in Fig. 1, for example, are
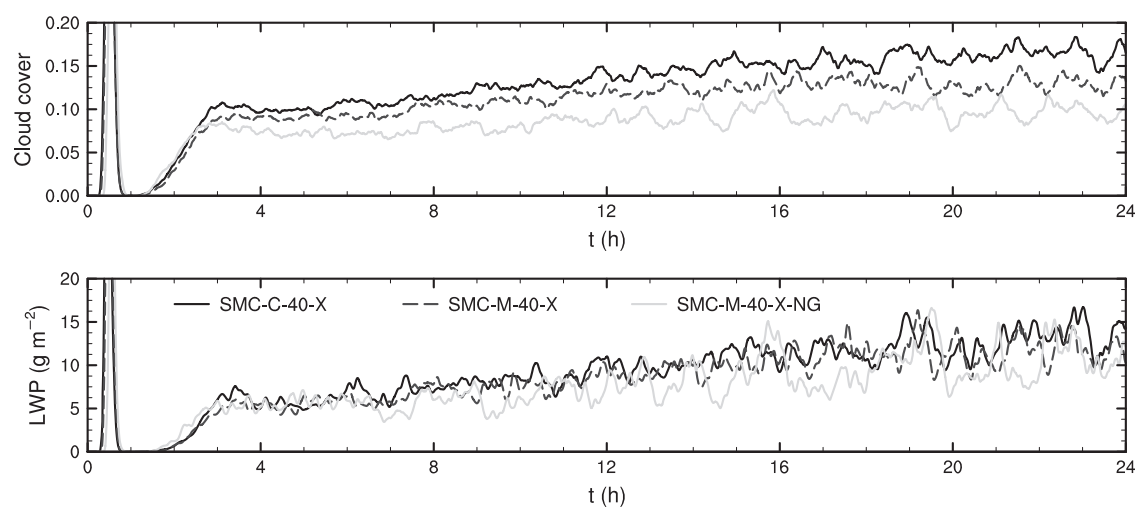

FIG. 4. Time evolution of cloud cover and LWP for the nonprecipitating cases with $\Delta x=40 \mathrm{~m}$. 


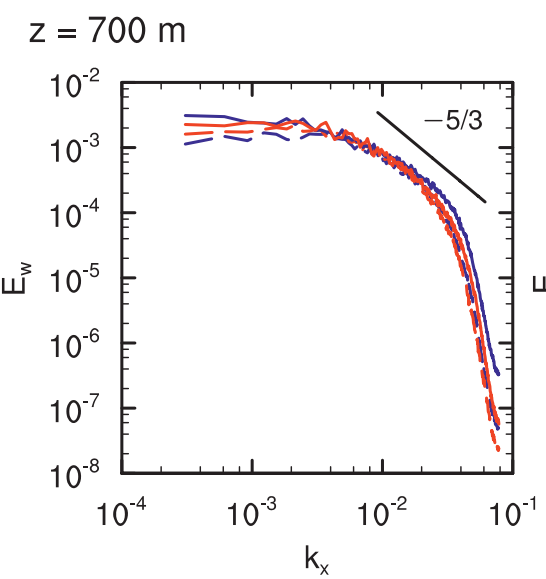

$z=1500 \mathrm{~m}$

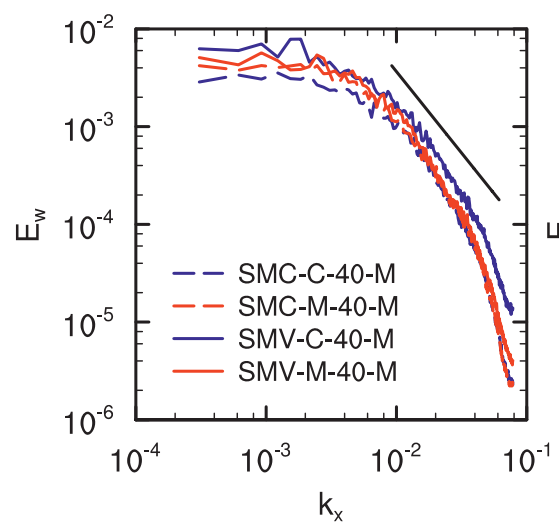

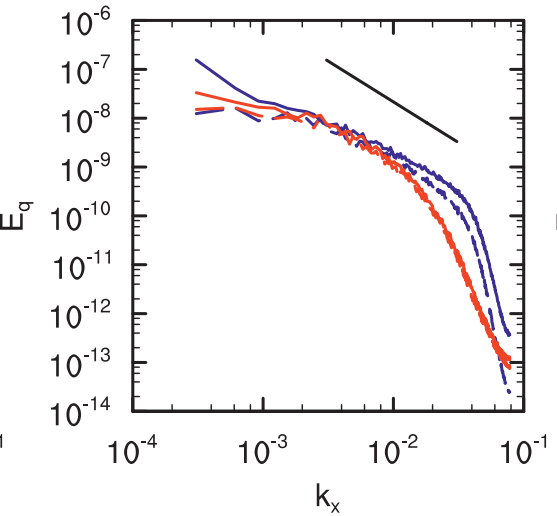
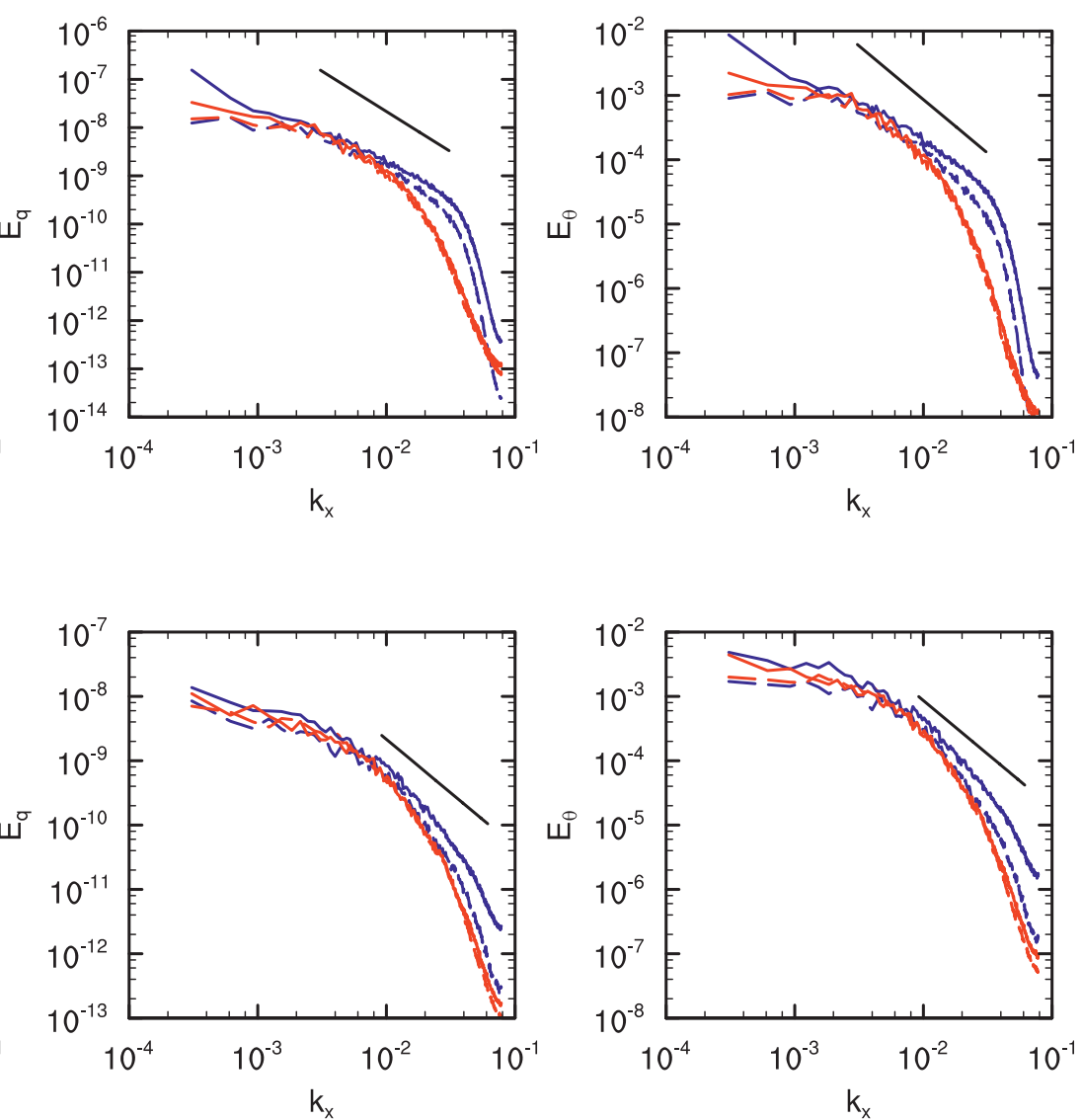

FIG. 5. One-dimensional power spectra along the zonal direction of (from left to right) vertical velocity, total water, and liquid water potential temperature for all runs with microphysics and $\Delta x=40 \mathrm{~m}$ at the end of the simulation, $t=24 \mathrm{~h}$, for (top) cloud base, $z=700 \mathrm{~m}$, and (bottom) about the middle of the cloud layer, $z=1500 \mathrm{~m}$.

meaningful and cannot be attributed to (small) random variations in the LES statistics.

The numerical dissipation introduced by the monotone scheme is expected to dampen high-wavenumber motions and can contribute to a decrease in cloud cover by diffusing small updrafts and low-amplitude fluctuations of humidity that could result in condensation. It is interesting that the effective dissipation can also change by altering the way the eddy diffusivity is computed as shown by the reduction in the vertically integrated turbulent kinetic energy (TKE) in Fig. 1. Momentum advection is always discretized with the nondissipative scheme; thus, the change in TKE is expected to be mostly attributed to the change of SGS implementation. Similar to the time evolution of cloud cover, liquid water path and TKE are less sensitive to numerical dissipation for implementation SMC. This suggests that SMC is more dissipative, resulting in "smoother" fields, a fact that reduces the amount of numerical dissipation. The monotone scheme approaches the nondissipative centered scheme when the flow fields contain negligible energy at high wavenumbers.

In contrast to simulations with SGS model implementation SMC, simulations with implementation SMV show initially no difference in TKE between the runs with and without numerical dissipation but diverge at later times. The increase in TKE is associated with a change in the character of convection. As shown in Fig. 2, clouds tend to organize in wind-parallel streaks except in case SMV-C-40-M where cloud clusters are aligned mainly in the wind-normal direction. The mean wind direction is along the diagonal in the panels of Fig. 2. This change in cloud organization is caused by precipitation. As shown in Fig. 1 and Table 2 the combination of implementation SMV with the nondissipative advection produces considerably more rain than the other combinations of SGS implementation and advection scheme.

Fortunately, the role of precipitation is easy to isolate, although this leads to a somewhat unphysical situation. 

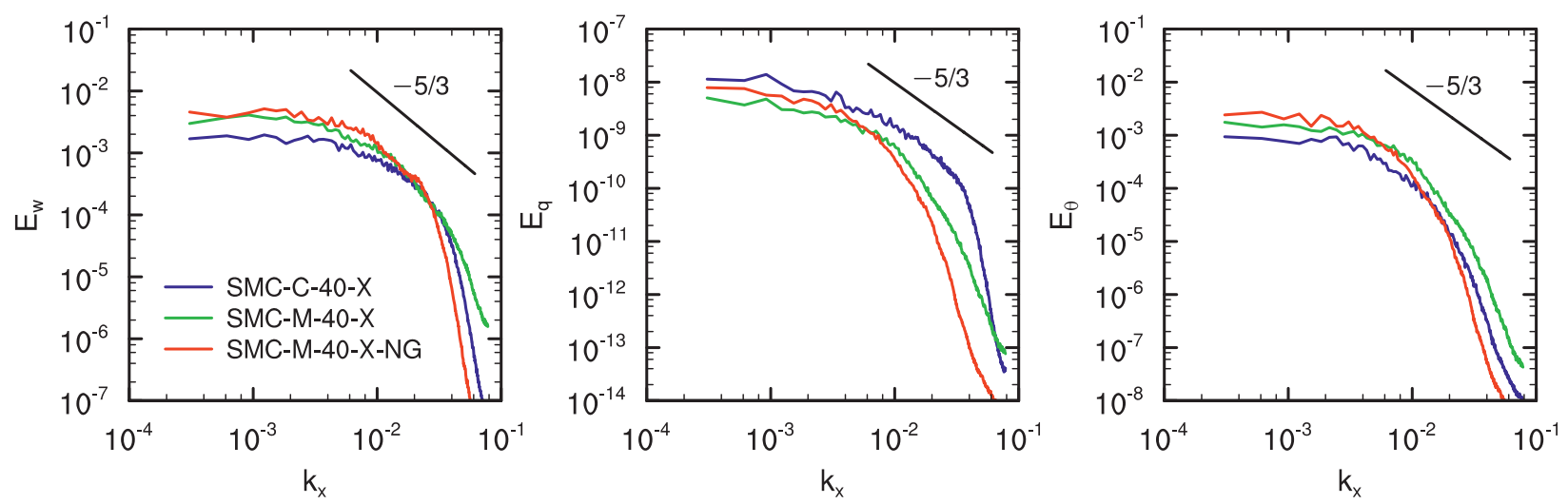

FIG. 6. Effects of increasing numerical dissipation on spectra for the nonprecipitating runs of Fig. 4 at $z=700 \mathrm{~m}$.

Figures 3 and 4 show results from runs without precipitation. These show the same trends with respect to cloud cover, LWP, and TKE as the precipitating cases: cloud cover, LWP, and TKE are higher for nondissipative scalar advection and SGS implementation SMV. Moreover, cloud organization does not change (Fig. 2) and differences in TKE traces are now small for SMV (Fig. 3). This corroborates the fact that the differences in TKE for $t>20 \mathrm{~h}$ and cloud organization for SMV are caused by the modification of the convection structure by precipitation. As shown in Table 2, the change of advection scheme for scalars can change the average surface precipitation rate during the last $4 \mathrm{~h}$ of the LES from 3.2 to $16.9 \mathrm{~W} \mathrm{~m}^{-2}$, resulting in a change in the convection structure and TKE.

The runs without precipitation show that the microphysical parameterization does not play a direct role in the observed differences and that these differences arise from the characteristics of the simulated turbulent fields. However, precipitation can amplify these differences. That is, runs without numerical dissipation and SGS model implementation SMV show more vigorous convection activity as suggested by increased levels of TKE and cloud cover. This results in the development of significant precipitation, which further alters the convection characteristics, causing additional divergence.

It is important to note that the addition of a translation velocity in the simulations reduces the absolute magnitude of the horizontal velocity field, thus reducing the numerical dissipation of the monotone scheme. As shown in Fig. 4 for a nonprecipitating case, the divergence of results between centered and monotone advection is larger if the translation velocity is not used. This is a notable disadvantage of this type of nonlinear monotone schemes as results depend on the computational frame of reference. The centered scheme and

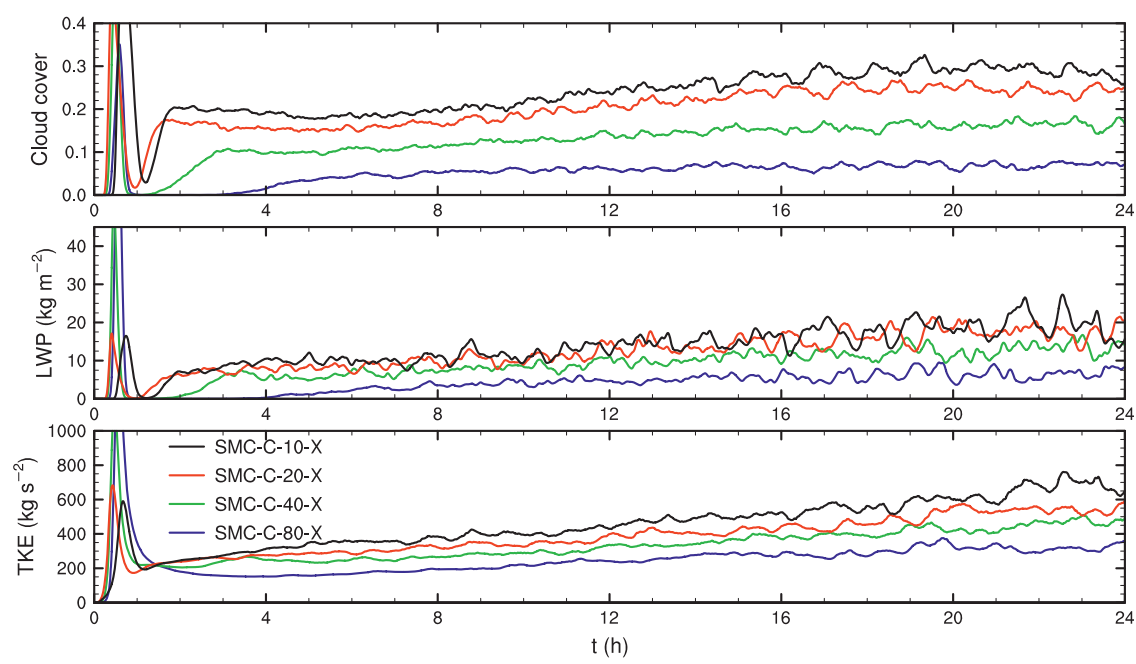

FIG. 7. Time evolution of cloud cover, LWP, and vertically integrated resolved-scale TKE for nonprecipitating runs at different resolutions. 

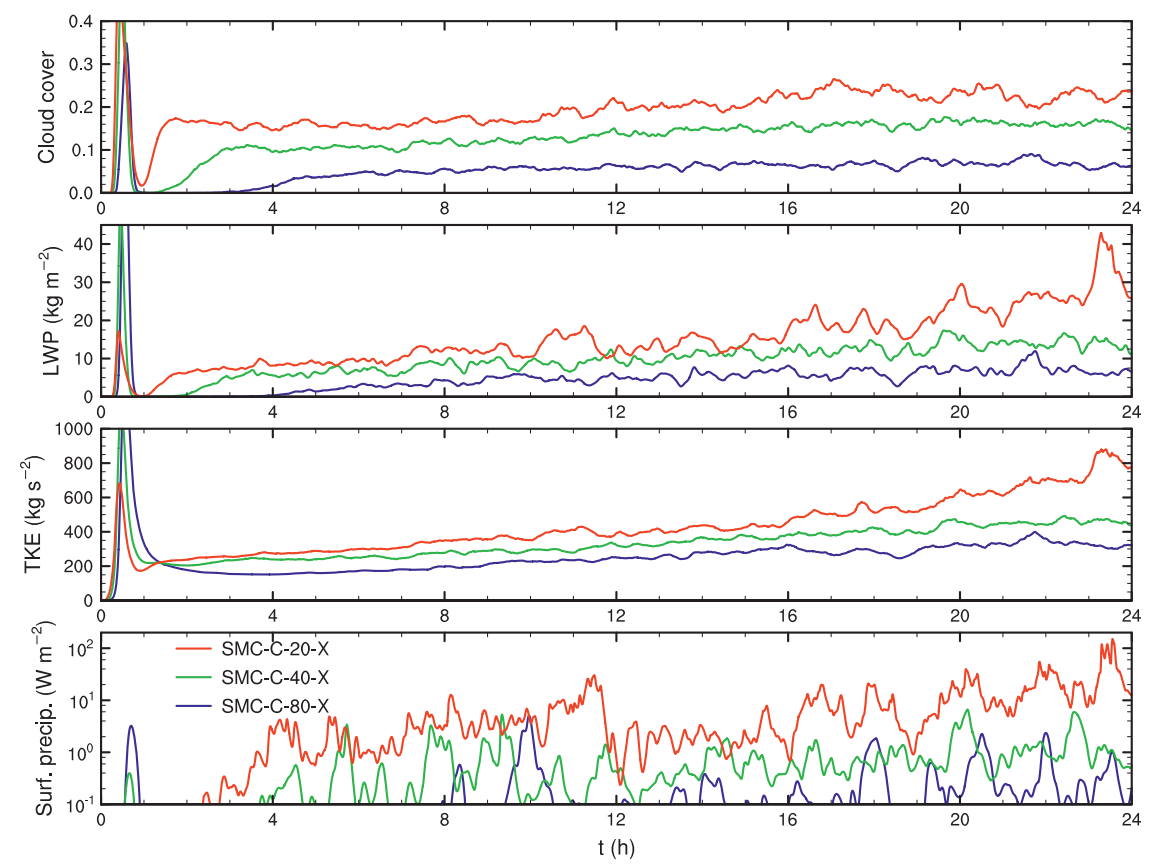

FIG. 8. Time evolution of cloud cover, LWP, vertically integrated resolved-scale TKE, and surface precipitation rate for precipitating runs at different resolutions.

both implementations of the SGS model are Galilean invariant.

Turbulence spectra are informative in revealing the average distribution of energy in the LES. Figure 5 shows one-dimensional power spectra of $w, q_{t}$, and $\theta_{l}$ along the zonal direction. Spectra along the meridional direction were found to be almost indistinguishable from the zonal ones despite the fact that the flow is not isotropic on horizontal planes, likely because of the symmetry of the flow along the diagonal of the domain (Fig. 2). Spectra are shown at two vertical levels, at about cloud base, $z=$ $700 \mathrm{~m}$, and the middle of the cloud layer, $z=1500 \mathrm{~m}$.

As expected, the imprint of numerical dissipation is noticeable in the shape of the spectra with the "rolloff" from near-power-law scaling occurring at smaller wavenumbers compared to the nondissipative scheme. Momentum is always advected with the nondissipative scheme and the vertical velocity spectrum appears not to be affected by the numerical dissipation of $q_{t}$ and $\theta_{l}$ advection.

The area between the spectra of $q_{t}$ and $\theta_{l}$ for simulations with and without numerical dissipation is proportional to the variance that is dissipated by the advection scheme. This amount of artificial dissipation appears to be larger at cloud base than in the cloud layer as shown in Fig. 5. Unlike cloud cover and TKE, spectra appear to be insensitive to SGS model implementation with numerical dissipation playing the dominant role at a given height. The effects of increasing numerical dissipation on the spectra are shown in Fig. 6 for nonprecipitating simulations. Dissipation significantly alters the energy distribution in the high wavenumbers while leaving low wavenumbers mostly unaffected.

\section{b. Grid resolution}

Grid resolution or the turbulence resolution scale is an important parameter in LES. Although predictions should not depend on the choice of grid spacing for sufficiently refined grids, often this is not the case. Four grid resolutions are employed $(\Delta x=10,20,40$, and $80 \mathrm{~m})$ for the nonprecipitating cases and three $(\Delta x=20$, 40 , and $80 \mathrm{~m}$ ) for the precipitating runs. To reduce the number of parameters and the effects of grid anisotropy, only uniform grids are used with $\Delta x=\Delta y=\Delta z$. As the grid is refined the domain size remains the same. The coarsest run has a grid size that is larger than vertical grid spacing used in most previous LES studies and this is expected to result in a decrease of accuracy. The finest grid results in a computationally expensive LES with a notable total number of grid cells: $2048^{2} \times 400=$ $1.67 \times 10^{9}$. For this reason, simulations are carried out using only the combination of SGS model implementation SMC and nondissipative scalar advection. This choice is motivated by the fact that SMC was found to be less sensitive. Moreover, the choice of nondissipative 
(a) $\Delta x=80 m$

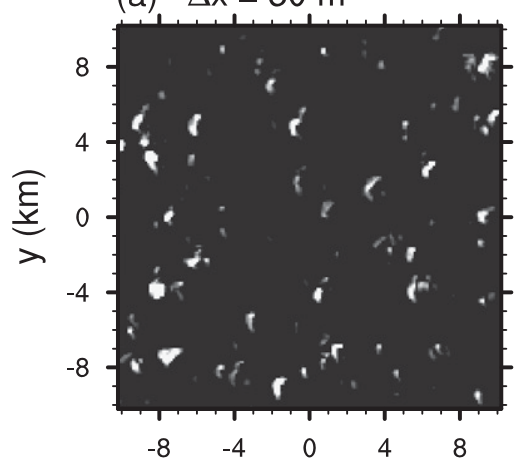

(b) $\Delta x=40 \mathrm{~m}$

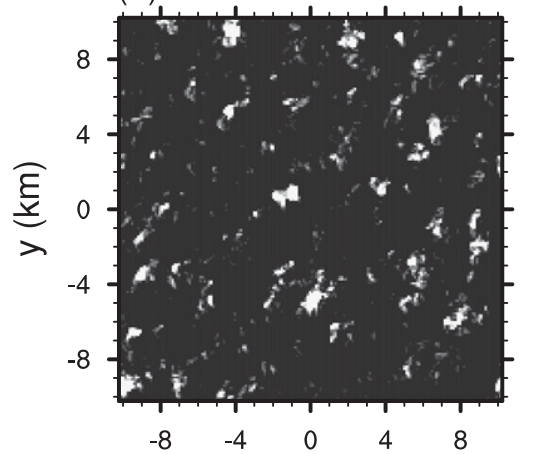

(c) $\Delta x=20 \mathrm{~m}$

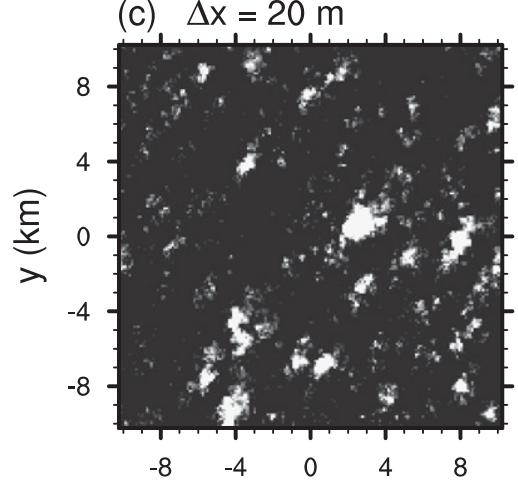

(d) $\Delta x=10 \mathrm{~m}$

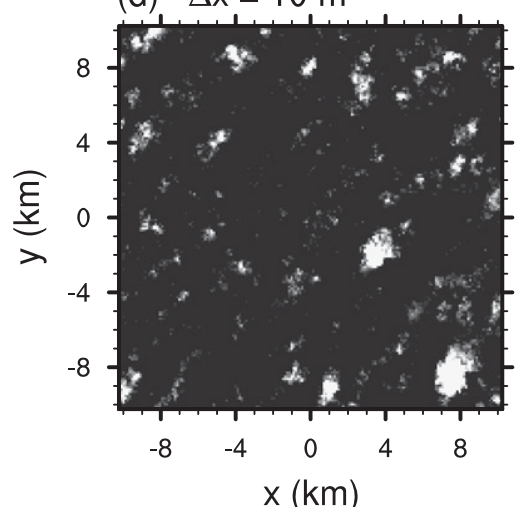

(e) $\Delta x=80 m$

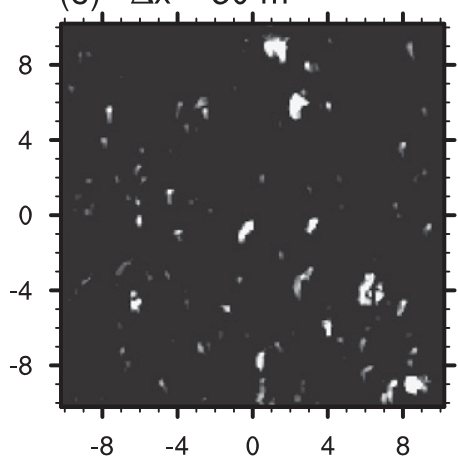

(f) $\Delta x=40 m$

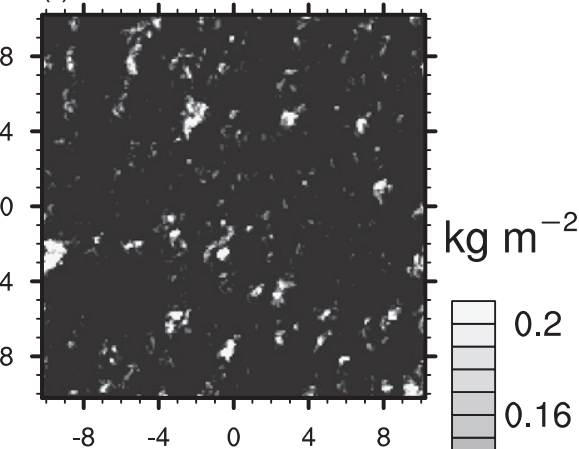

(g) $\Delta x=20 m$

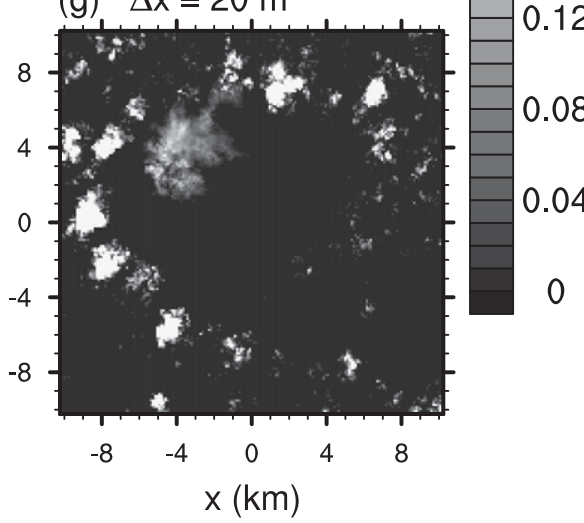

FIG. 9. Cloud LWP at the end of the simulation, $t=24 \mathrm{~h}$, for runs with varying grid resolution. Left column corresponds to simulations without precipitation. 
TABLE 3. Average surface precipitation rate, cloud cover (CC), LWP, and vertically integrated TKE during the last $4 \mathrm{~h}(t=20$ $24 \mathrm{~h}$ ) of the simulations with varying resolution.

\begin{tabular}{lccccc}
\hline \hline Case & $\begin{array}{c}\Delta x \\
(\mathrm{~m})\end{array}$ & $\begin{array}{c}P_{\text {srf }} \\
\left(\mathrm{W} \mathrm{m}^{-2}\right)\end{array}$ & \multicolumn{1}{c}{ C } & $\begin{array}{c}\mathrm{LWP} \\
\left(\mathrm{g} \mathrm{m}^{-2}\right)\end{array}$ & $\begin{array}{c}\mathrm{TKE} \\
\left(\mathrm{kg} \mathrm{s}^{-2}\right)\end{array}$ \\
\hline SMC-C-80-M & 80 & 0.5 & 0.07 & 7.1 & 332 \\
SMC-C-40-M & 40 & 1.5 & 0.16 & 13.9 & 453 \\
SMC-C-20-M & 20 & 25.1 & 0.23 & 27.0 & 705 \\
SMC-C-80-X & 80 & - & 0.07 & 6.4 & 315 \\
SMC-C-40-X & 40 & - & 0.17 & 1.1 & 451 \\
SMC-C-20-X & 20 & - & 0.24 & 17.7 & 541 \\
SMC-C-10-X & 10 & - & 0.29 & 19.3 & 659 \\
\hline
\end{tabular}

advection has the advantage of being unambiguous when comparing with other studies as there are several ways a dissipative or monotone scheme can be formulated. The coefficients in the parameterization of the surface fluxes are the same for all grid resolutions although their values strictly correspond to the $\Delta x=40 \mathrm{~m}$ case. For all resolutions surface fluxes are virtually the same. For instance, the difference in mean latent heat flux between $\Delta x=40 \mathrm{~m}$ and $\Delta x=20 \mathrm{~m}$ simulations is about $2 \%$.

Significant differences in the evolution of the boundary layer are observed by varying the grid resolution. Cloud cover appears to be the most sensitive quantity and, as shown in Figs. 7 and 8, increases with decreasing grid spacing, from about $7 \%$ for $\Delta x=80 \mathrm{~m}$ to about $25 \%$ for $\Delta x=20 \mathrm{~m}$, almost a factor of 4 increase. For all resolutions, precipitation appears to have almost no effect on cloud cover.

Some increase in cloud cover is expected with decreasing grid spacing as smaller clouds are allowed to form when using finer grids. However, as shown in Fig. 9, the greater part of the increase in cloud cover with respect to grid resolution comes from the fact that clouds get larger. A second important observation is that the increase in cloud sizes is accompanied with a change in the cloud organization, for both precipitating and nonprecipitating cases. This is particularly striking for the precipitating case where clouds organized in wind-parallel streaks change into arcs.

Similar to the observed effects of reducing the numerical dissipation, increasing grid resolution leads to more precipitation, which changes the convection structure. As shown in Table 3 and Fig. 8, surface precipitation rates exhibit strong sensitivity to grid resolution.

Figure 10 shows the three-dimensional cloud structure for the high-resolution precipitating and nonprecipitating cases. Precipitation causes the cloud structure to change from streaks to arcs surrounding cold pools with remnant thin high clouds at the center (see also Fig. 9). The simulated cloud structure and variability (Fig. 10) are quite realistic and are in qualitative agreement with observations during RICO (Snodgrass et al. 2009). The high-resolution
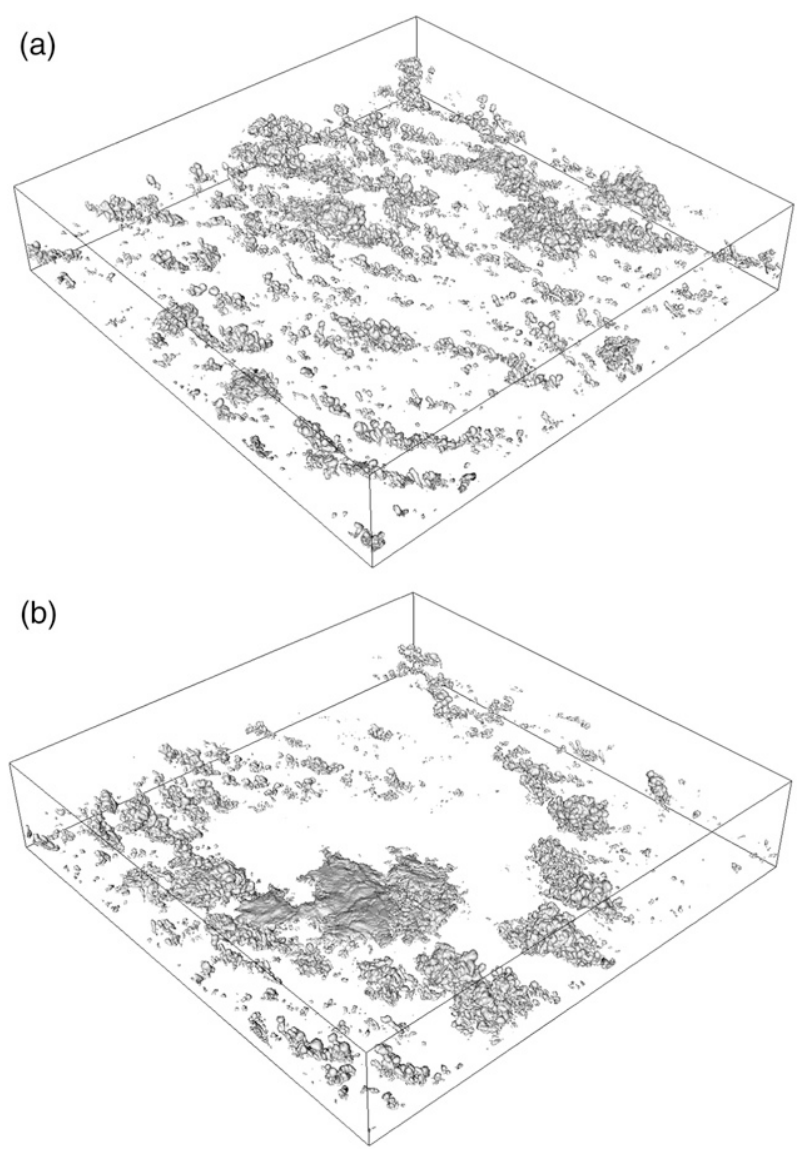

FIG. 10. Rendering of cloud liquid water corresponding to isosurface $q_{c}=1 \times 10^{-5}$ for the (a) nonprecipitating and (b) precipitating cases, at the end of the simulation, $t=24 \mathrm{~h} ; \Delta x=20 \mathrm{~m}$, nondissipative advection, with subgrid model implementation SMC. Cloud LWP is plotted in Figs. 9c,g.

precipitating case indicates that the domain might be small because a single convection complex fills the entire domain and could be constrained by the (arbitrarily) chosen domain size.

Vertical profiles for different resolutions are shown in Fig. 11. The profiles were computed using a space-time average over the last $4 \mathrm{~h}$ of the simulation. Mean quantities are largely insensitive to changes in resolution except the profile of cloud liquid water, which shows differences in the amount of liquid and its vertical distribution up to $\Delta x=20 \mathrm{~m}$. Mean wind, potential temperature, and total water profiles were also found to be insensitive to modeling choices in model intercomparison studies (e.g., Siebesma et al. 2003; VanZanten et al. 2011). This is a consequence of the lateral periodic boundary condition, which imposes severe constraints on the evolution of the mean quantities. The mean is mostly driven by the boundary and large-scale forcings and to a much lesser extent by small-scale turbulence. 
(a)
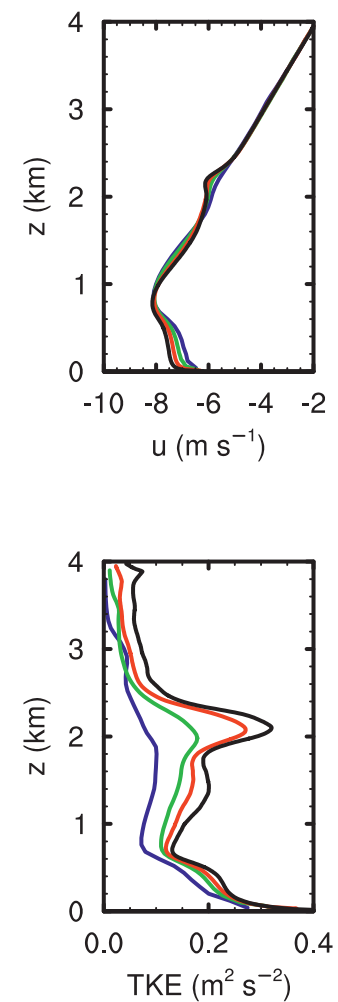

(b)
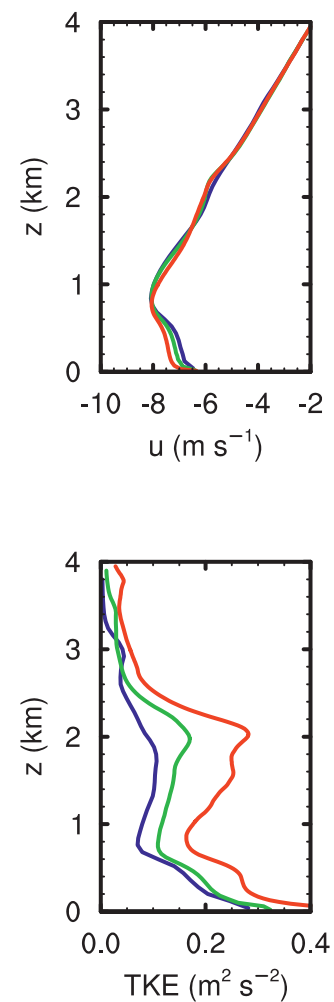
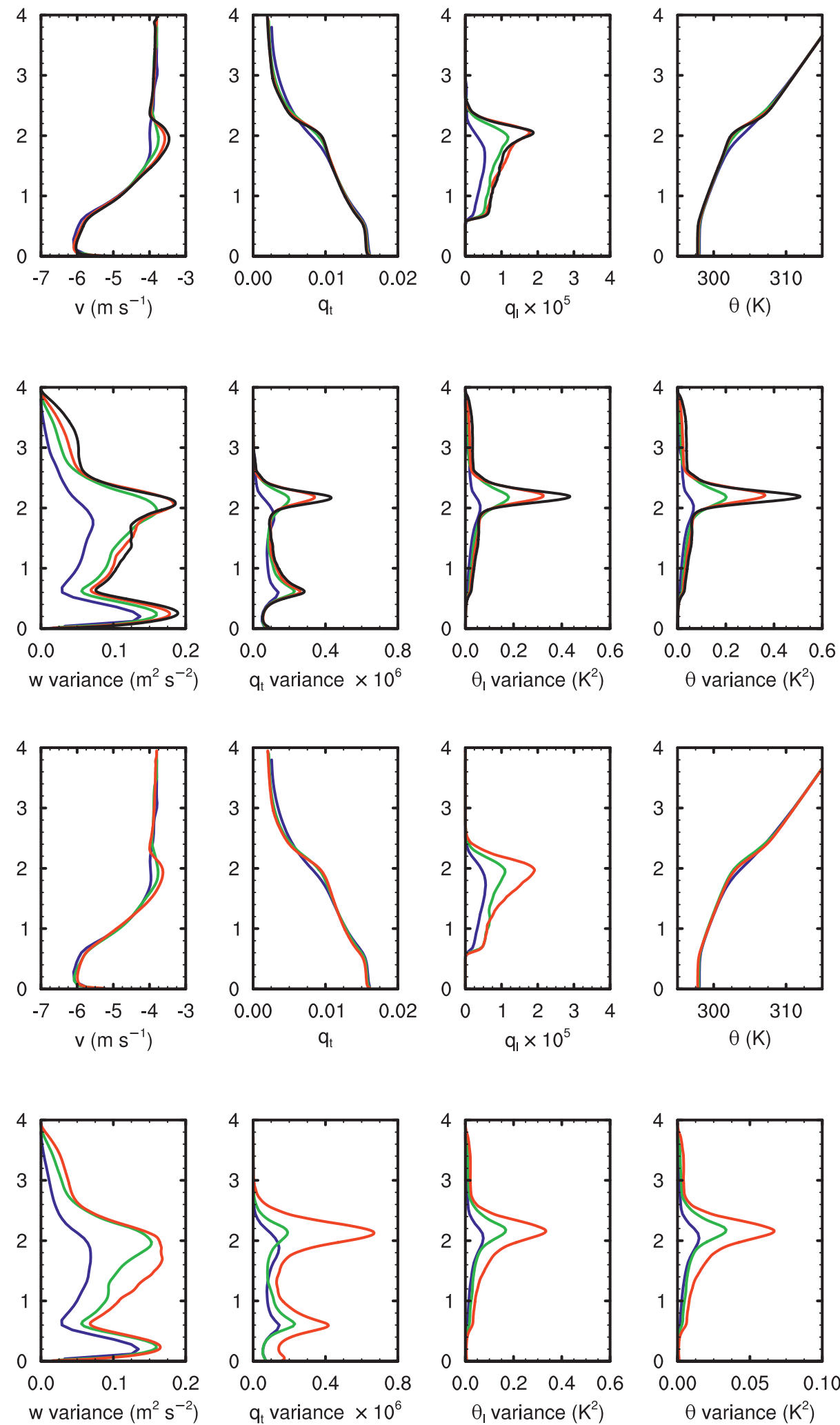

FIG. 11. Vertical profiles for runs with different resolutions. Top panels correspond to the nonprecipitating case. Line colors are as in Fig. 7. 

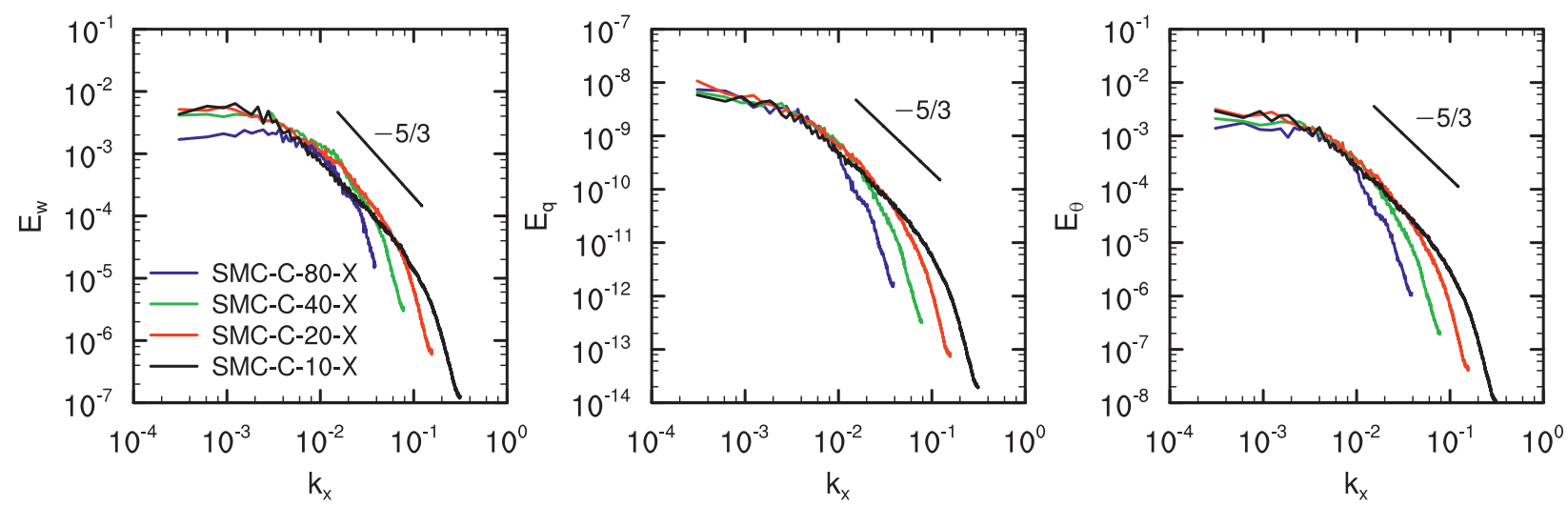

FIG. 12. One-dimensional power spectra along the zonal direction of vertical velocity, total water, and liquid water potential temperature at $z=1500 \mathrm{~m}$.

Second-order moments show larger variations with respect to resolution. However, in the nonprecipitating case there is good agreement between the two highest resolutions with most of the differences confined near the inversion. Figures 7 and 11 indicate that resolution independence is achieved for the nonprecipitating cases for $\Delta x=20 \mathrm{~m}$.

Figure 12 shows spectra for the nonprecipitating cases for the four resolutions used. Only the highestresolution run appears to indicate a nearly constant power-law scaling, especially for the spectrum of vertical velocity. For all resolutions fluctuations at the highest wavenumbers are dumped on average by the SGS model (Skamarock 2004).

\section{Discussion}

The present study investigates the performance of large-eddy simulation in a case of shallow precipitating cumulus convection. The current simulations demonstrate that when using a typical turbulence closure and numerical method, the results show significant sensitivities with respect to the way the discrete problem is formulated. Although this is justified to some extent by the complexity of the flow, it is intriguing that significant sensitivities were not observed in simpler flows (e.g., Nieuwstadt et al. 1993). This leads to the question of how the present code will perform on a much simpler flow and whether the differences could have been anticipated.

Four simulations of a dry convective boundary layer were carried out to assess the performance of the current LES code for a simpler flow and answer the aforementioned question. The LES runs utilize the two model implementations, SMC and SMV, and two grid resolutions, $\Delta x=20$ and $40 \mathrm{~m}$. The nondissipative advection scheme is used for all runs. The domain size is $10.24^{2} \times$ $4 \mathrm{~km}^{3}$. The potential temperature lapse rate is $2 \mathrm{~K} \mathrm{~km}^{-1}$, with $\theta(z=0)=297 \mathrm{~K}$. The total water mixing ratio lapse rate is $-0.37 \times 10^{-3} \mathrm{~km}^{-1}$ up to $z=1350 \mathrm{~m}$ and $-0.94 \times$ $10^{-3} \mathrm{~km}^{-1}$ higher up with $q_{t}(z=0)=5 \times 10^{-3}$. The temperature and humidity surface fluxes are $0.06 \mathrm{~K} \mathrm{~m} \mathrm{~s}^{-1}$ and $2.5 \times 10^{-5} \mathrm{~m} \mathrm{~s}^{-1}$, respectively.

Figures 13 and 14 show that the differences between all cases are negligible. Figure 13 shows the normalized growth of the boundary layer, using the minimum of the buoyancy flux as the definition of the inversion height $z_{i}$. The profiles of Fig. 14 are computed by averaging the corresponding instantaneous fields at $t=8 \mathrm{~h}$ (no time averaging). The inversion height is normalized using the encroachment growth $z_{e}$ (Stull 1988, p. 666). The growth rate is in agreement with earlier studies (e.g., Siebesma

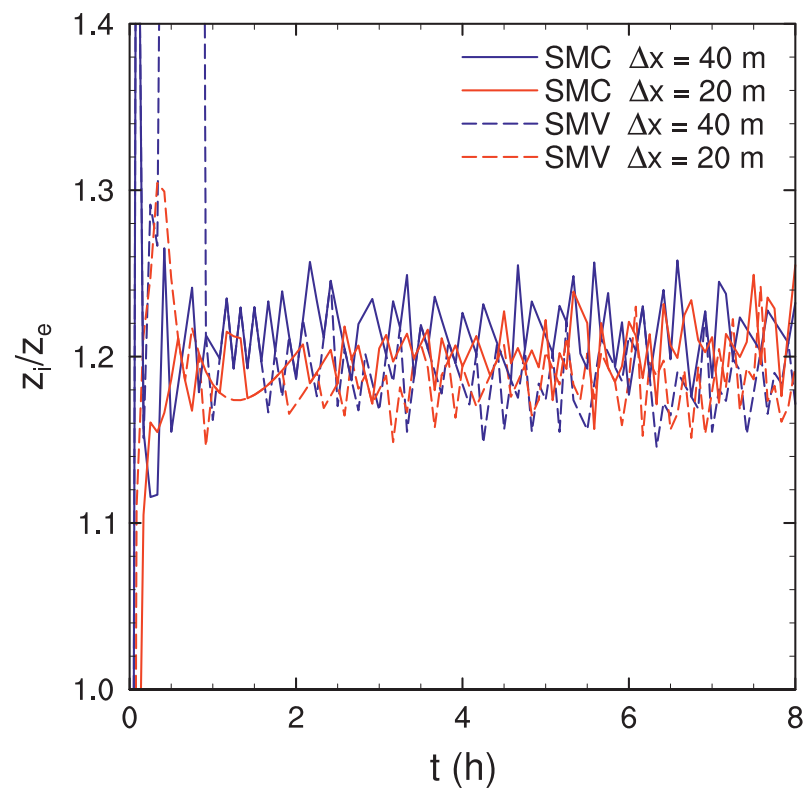

FIG. 13. Growth rate of a dry convective boundary layer computed with the two subgrid model implementations at two resolutions each. 

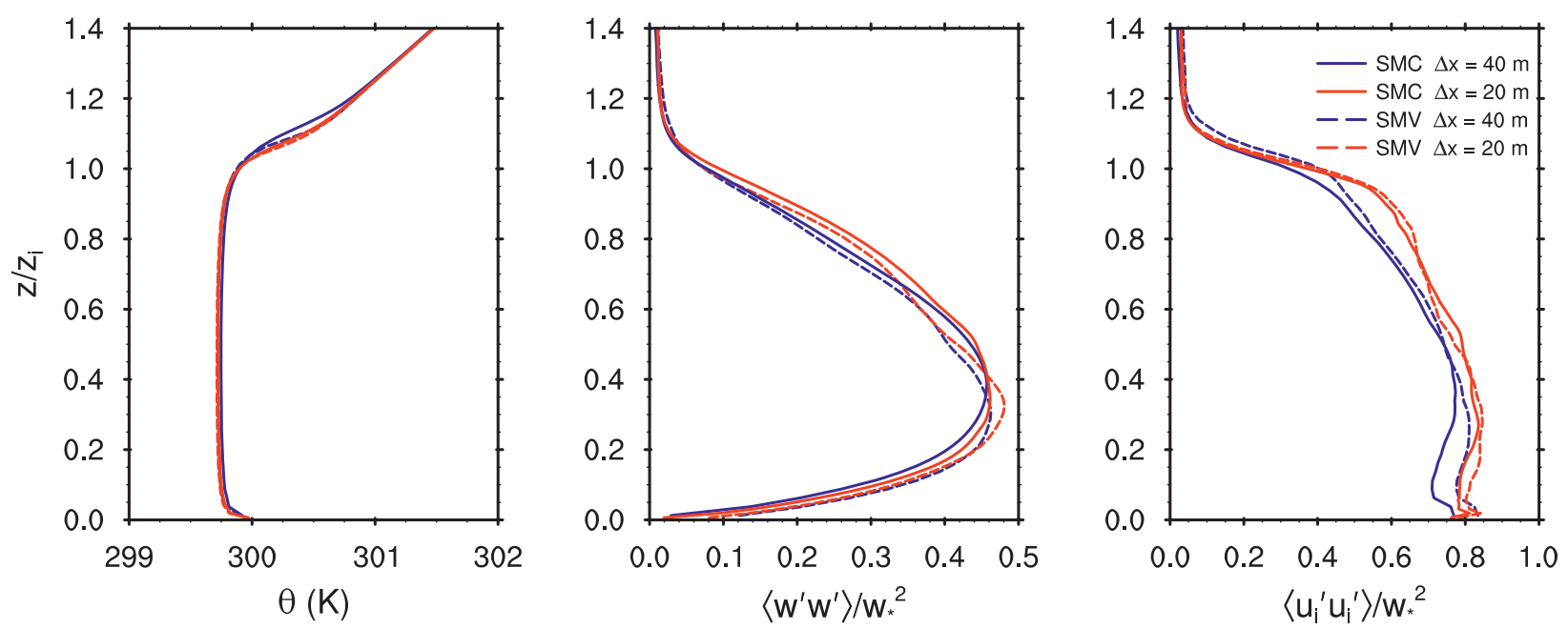

FIG. 14. Mean profiles for dry convective boundary layer runs with the two subgrid model implementations at two resolutions, 20 and $40 \mathrm{~m}$. From left to right: potential temperature, vertical velocity variance, and resolved-scale TKE. The top of the computational domain is at $z / z_{i}=2.5$.

et al. 2007). Figure 14 shows vertical profiles at $t=8 \mathrm{~h}$. Mean potential temperature and vertical velocity variance are in very good agreement. TKE profiles show larger differences but they are mainly a result of the change in resolution rather than the model implementation.

The dry convective boundary layer results support the initial hypothesis that the observed differences in the present shallow cumulus convection LES are attributable to the increased difficulty of modeling this case. This difficulty emerges from the amplified nonlinearity in the two-phase flow. Figure 15 shows horizontal slices of potential temperature for two resolutions of the dry boundary layer LES. When the resolution is doubled the overall structure of the potential temperature field remains unchanged, but the higher-resolution LES resolves finer motions. In this case, the potential temperature is essentially buoyancy, which is the physical quantity that drives the turbulence in this flow. A comparison of the buoyancy field for the cumulus convection LES, shown in Fig. 16, displays considerably different behavior. The buoyancy field-in other words, a main force driving the flow-is dominated by the process of latent heat exchange. Because of evaporative cooling, the cloud edges are negatively buoyant (Heus and Jonker
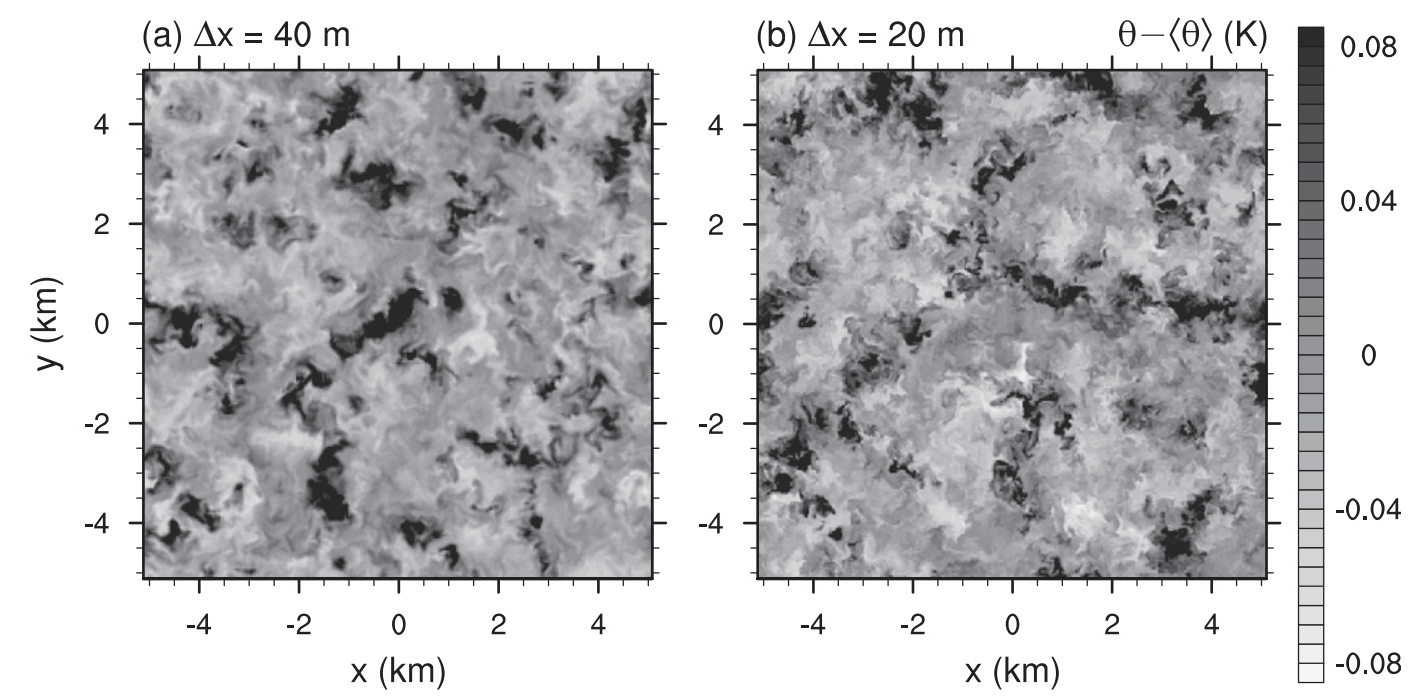

FIG. 15. Instantaneous potential temperature perturbation $(\theta-\langle\theta\rangle)$ at half height of a dry convective boundary layer LES at two resolutions. 
(a) SMC-C-40-X

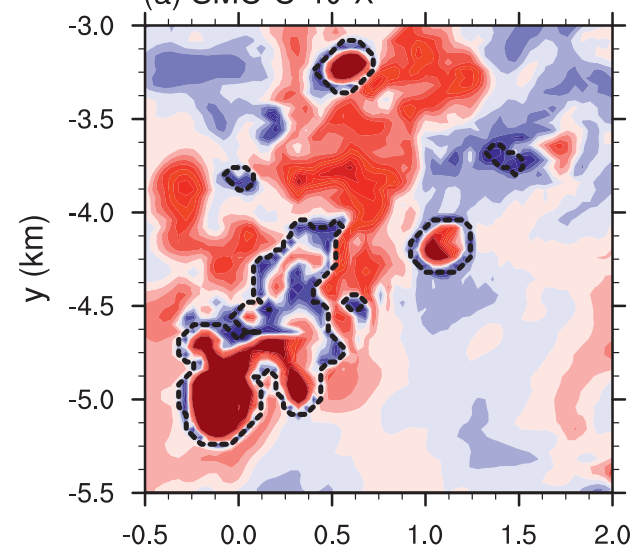

(c) SMC-C-20-X

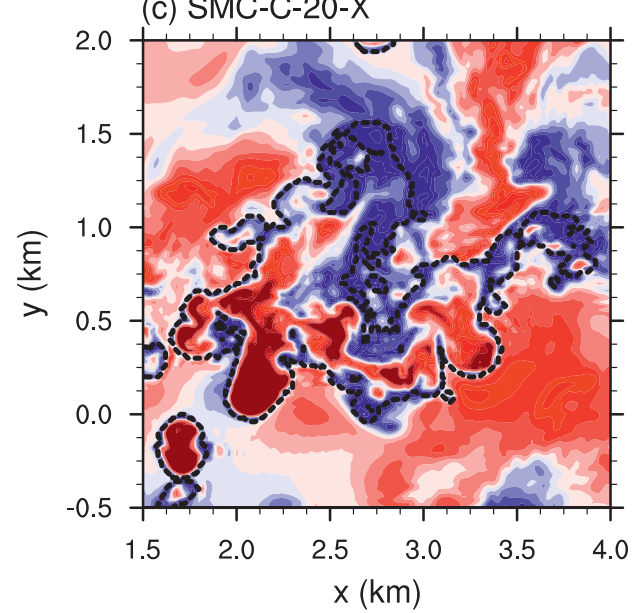

(b) SMC-C-40-X

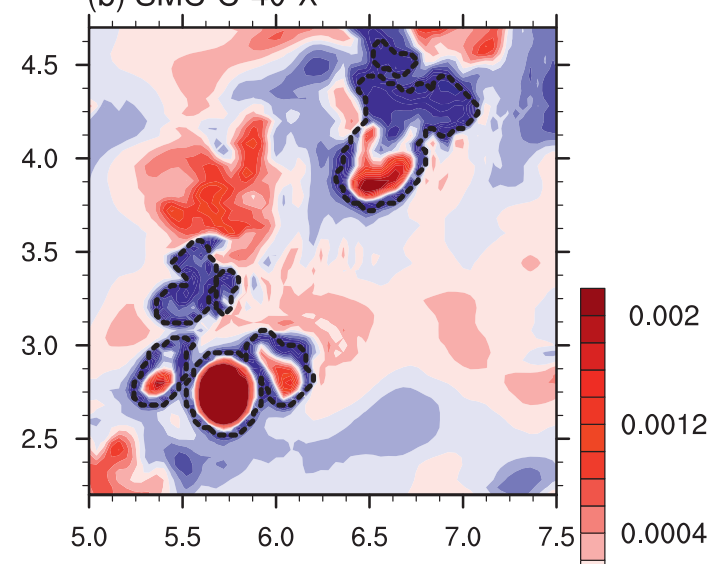

(d) SMC-C-20-X

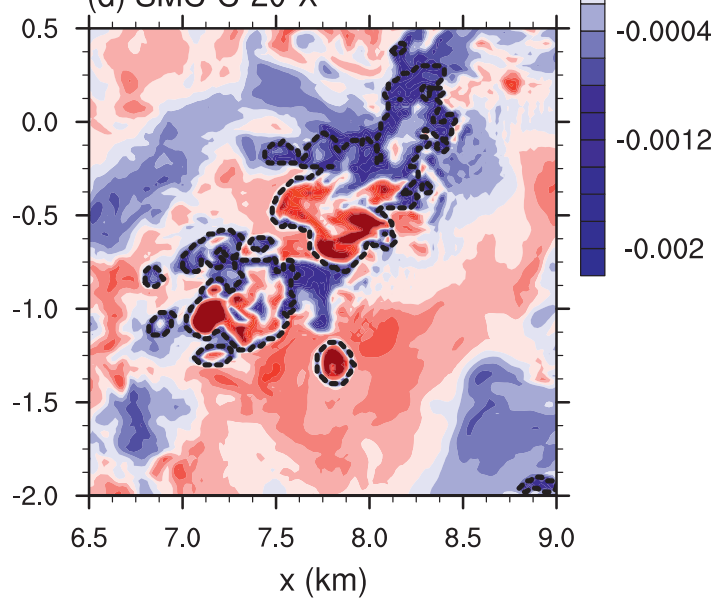

FIG. 16. Buoyancy at $z=1500 \mathrm{~m}$ for nonprecipitating cumulus convection cases at (top) medium resolution, $\Delta x=$ $40 \mathrm{~m}$, and (bottom) high resolution, $\Delta x=20 \mathrm{~m}$. Black dotted contour corresponds to the saturation surface. Each panel shows a $2.5 \times 2.5 \mathrm{~km}^{2}$ subsection of the computational domain at $t=24 \mathrm{~h}$. Note the thin negative buoyancy (dark blue contour) surface surrounding the clouds.

2008). The negative buoyancy surface is unresolved for typical LES grid resolutions and therefore scales with the grid spacing, unlike the convective structures of the dry case.

LES-type models are particularly suitable for the prediction of flows that are nearly homogeneous, such as the dry case shown in Fig. 15-a fact corroborated by the present results. The moist case stresses the scalesimilarity assumption, which likely leads to the observed sensitivities. Moreover, the negative buoyancy surfaces occur at the important interface between cloud and environment, thus possibly exacerbating the modeling difficulty of capturing the entrainment process at cloud boundaries. Use of a condensation scheme that takes into account the subgrid distribution of temperature and humidity (Sommeria and Deardorff 1977) instead of the all-or-nothing scheme used here may alleviate the problem. The all-or-nothing scheme does not appear to set a (thickness) length scale for the negative buoyancy surfaces.

The choice of advection scheme was found to significantly affect the LES prediction, especially the precipitation rates. This mainly arises from the introduction of numerical dissipation in monotone advection schemes in order to control spurious dispersive spatial oscillations. Figure 17 shows a horizontal slice of total water for case SMC-C-20-X (nondissipative advection). A careful examination of Fig. 17 discloses short wavelength oscillations (about $5 \Delta x$ ) in a few places downwind of the clouds (for the three-point stencil centered-difference scheme used here, shorter wavelengths propagate more slowly than longer wavelengths). The contours of Fig. 17 were carefully adjusted to make the oscillations visible as the amplitude of the oscillations is only about $5 \%$ of the 


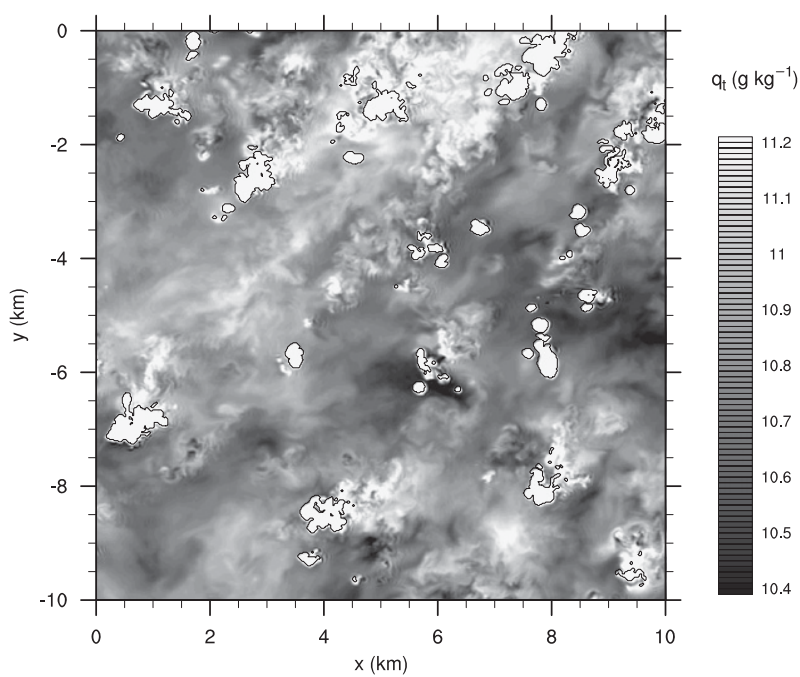

FIG. 17. Total water mixing ratio (grayscale contours) for case SMC-C-20-X on a horizontal plane at $z=1500 \mathrm{~m}$, about the middle of the cloud layer. Only a quarter of the computational domain is shown. Black contours denote the saturation mixing ratio. Small-amplitude dispersive oscillations are discernible downwind (northeast) of the clouds [e.g., at $(6,-4)$ and $(9,-5)$ $\mathrm{km}]$. The maximum and minimum of $q_{t}$ on the entire plane are, 15.9 and $9.7 \mathrm{~g} \mathrm{~kg}^{-1}$.

range of $q_{t}$ on the entire plane; thus, in this case of shallow convection the adverse effects of the nondissipative scheme are minor and are compensated by the advantages of conservation of kinetic energy and scalar variance.

Although the resolution and domain size used in the present investigation are larger than the GCSS RICO case study (VanZanten et al. 2011), results show that LES statistics converged with respect to resolution only for very fine grids. Typical resolution arguments that are based on the ratio of unresolved to total TKE appear to be less appropriate for this multiscale flow since updrafts and clouds set important length scales that need to be adequately resolved. Also, in simulations of deep convection, Bryan et al. (2003) argue that resolution should be fine enough to resolve at least a part of the inertial range. The current simulations show that even with an inertial range spanning almost two decades (Fig. 12), LES low-order statistics, such as mean liquid water, are not converged.

Moreover, difficulties emerge in the largest scales of the flow. The precipitating simulations exhibit significant mesoscale organization that has to be captured in order to accurately simulate the evolution of the individual cloud elements. The present simulations show that a domain of $20 \times 20 \mathrm{~km}^{2}$ may not be sufficiently large to include important large scales.

\section{Conclusions}

The main implication of the current investigation is that large-eddy simulation (LES) predictions of cumulus convection can exhibit significant dependence on the discrete representation of the governing equations. Differences in LES predictions have been documented by previous model intercomparison studies (e.g., Siebesma et al. 2003; Stevens et al. 2005); however, the current study provides deeper insight into model choices and their connection to the flow physics by meticulously controlling for various model parameters.

The increased sensitivity of the two-phase flow to modeling choices is illustrated by the observed differences when using two different discretizations of the same subgrid-scale model. This type of difference is not apparent when a single-phase flow, a dry convective boundary layer, is simulated.

For the nonprecipitating cases grid convergence is achieved for grid resolutions of about $20 \mathrm{~m}$. This conclusion cannot be extrapolated to precipitating cumulus because these cases were found to be more sensitive to model configuration. For instance, low-resolution simulations have low cloud cover with clouds organized in wind-parallel streaks, whereas high-resolution runs with higher cloud cover develop significant precipitation that results in clouds organized in arcs surrounding cold pools. In this case, a single cold pool complex fills the entire computational domain, suggesting that computational domains of $20 \times 20 \mathrm{~km}^{2}$ may be too small to accurately simulate shallow precipitating convection.

Cloud fraction was found to be the most sensitive parameter with respect to resolution and changes in cloud fraction are observed even when the mean liquid water has converged. Therefore, its usefulness in comparing LES results can be limited.

Acknowledgments. We would like to acknowledge many fruitful discussions with Paul Dimotakis and Pier Siebesma. JT and GM acknowledge the support provided by the Office of Naval Research, Marine Meteorology Program under Award N0001408IP20064, and by the NASA MAP Program. Resources supporting this work were provided by the NASA High-End Computing (HEC) Program through the NASA Advanced Supercomputing (NAS) Division at Ames Research Center. Simulations were also performed on the Shared Heterogeneous Cluster at the Center for Advanced Computing Research (CACR) at Caltech developed with cofunding by NSF MRI Grant EIA-0079871 and AFOSR Grant FA9550-07-1-0091 as part of a larger research effort on turbulent mixing. This research was carried out at the Jet Propulsion Laboratory, California 

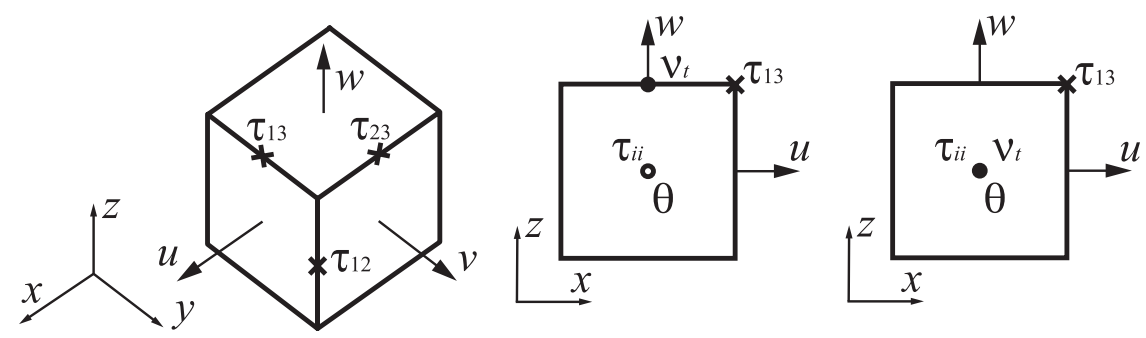

FIG. A1. (left) Variable arrangement on the computational grid. Scalar variables are denoted by $\theta$ and the eddy diffusivity by $\nu_{t}$. (middle) Model SMV configuration; (right) model SMC.

Institute of Technology, under a contract with the National Aeronautics and Space Administration.

\section{APPENDIX A}

\section{Details of the Discrete Representation of the SGS Tensor}

The two formulations of the Smagorinsky SGS model differ in the location where the eddy diffusivity is defined and how the deformation is computed. In implementation SMC, the eddy diffusivity is defined at cell centers whereas in SMV is computed at the vertical-face centers, the same location as the vertical velocity. In both cases the elements of the SGS tensor are computed at their natural locations. The diagonal terms are computed at the cell centers and the off-diagonal terms in the middle of the cell edges as shown in Fig. A1. For the off-diagonal SGS tensor elements the eddy viscosity is interpolated at these locations.

Difference and interpolation operators are constructed mainly following the notation of AntonopoulosDomis (1981). Uniform grid spacing is assumed in all subsequent expressions. A family of central difference operators $\delta$ is defined. For instance,

$$
\delta_{1} \phi(l, m, n) \equiv \frac{1}{\Delta x_{1}}\left[\phi\left(l-\frac{1}{2}, m, n\right)+\phi\left(l+\frac{1}{2}, m, n\right)\right]
$$

denotes the central midpoint difference of variable $\phi$ evaluated at $(l, m, n)$ along direction 1 . The usual secondorder central difference operator is

$$
\delta_{1(2)} \phi(l, m, n) \equiv \frac{1}{2 \Delta x_{1}}[\phi(l-1, m, n)+\phi(l+1, m, n)] .
$$

Two interpolation operators are also defined, a onedimensional interpolation,

$$
\alpha_{1} \phi(l, m, n) \equiv \frac{1}{2}\left[\phi\left(l-\frac{1}{2}, m, n\right)+\phi\left(l+\frac{1}{2}, m, n\right)\right],
$$

and a two-dimensional interpolation at cell centers, written here on a horizontal plane:

$$
\begin{aligned}
\alpha_{3}^{\square} \phi(l, m, n) \equiv & \frac{1}{4}\left[\phi\left(l-\frac{1}{2}, m-\frac{1}{2}, n\right)+\phi\left(l-\frac{1}{2}, m+\frac{1}{2}, n\right)\right. \\
& \left.+\phi\left(l+\frac{1}{2}, m-\frac{1}{2}, n\right)+\phi\left(l+\frac{1}{2}, m+\frac{1}{2}, n\right)\right] .
\end{aligned}
$$

\section{a. Subgrid-scale model implementation SMC}

In SGS model implementation SMC, approximations of the components of the rate of strain tensor $D_{i j}$ are computed at cell centers. The two types of velocity derivatives required are approximated by

$$
\frac{\partial u_{i}}{\partial x_{i}}(l, m, n) \approx \delta_{i} u_{i}+\mathcal{O}\left(\Delta x_{i}^{2}\right)
$$

and

$$
\frac{\partial u_{i}}{\partial x_{j}}(l, m, n) \approx \alpha_{i}\left[\delta_{j(2)} u_{i}\right]+\mathcal{O}\left(\Delta x_{j}^{2}\right)
$$

\section{b. Subgrid-scale model implementation SMV}

In implementation SMV, first the squares $D_{i j} D_{i j}$ are computed at the cell edges and then are interpolated at the $w$ locations:

$$
\begin{aligned}
D_{i j} D_{i j}\left(l, m, n+\frac{1}{2}\right)= & \alpha_{3} D_{i i}^{2}+2\left[\alpha_{1} D_{13}^{2}+\alpha_{2} D_{23}^{2}\right. \\
& \left.+\alpha_{3}\left(\alpha_{3}^{\square} D_{12}^{2}\right)\right] .
\end{aligned}
$$

The velocity gradients for the elements of $D_{i j}$ are evaluated at the locations of $\tau_{i j}$ (see Fig. A1) using centered differences: 

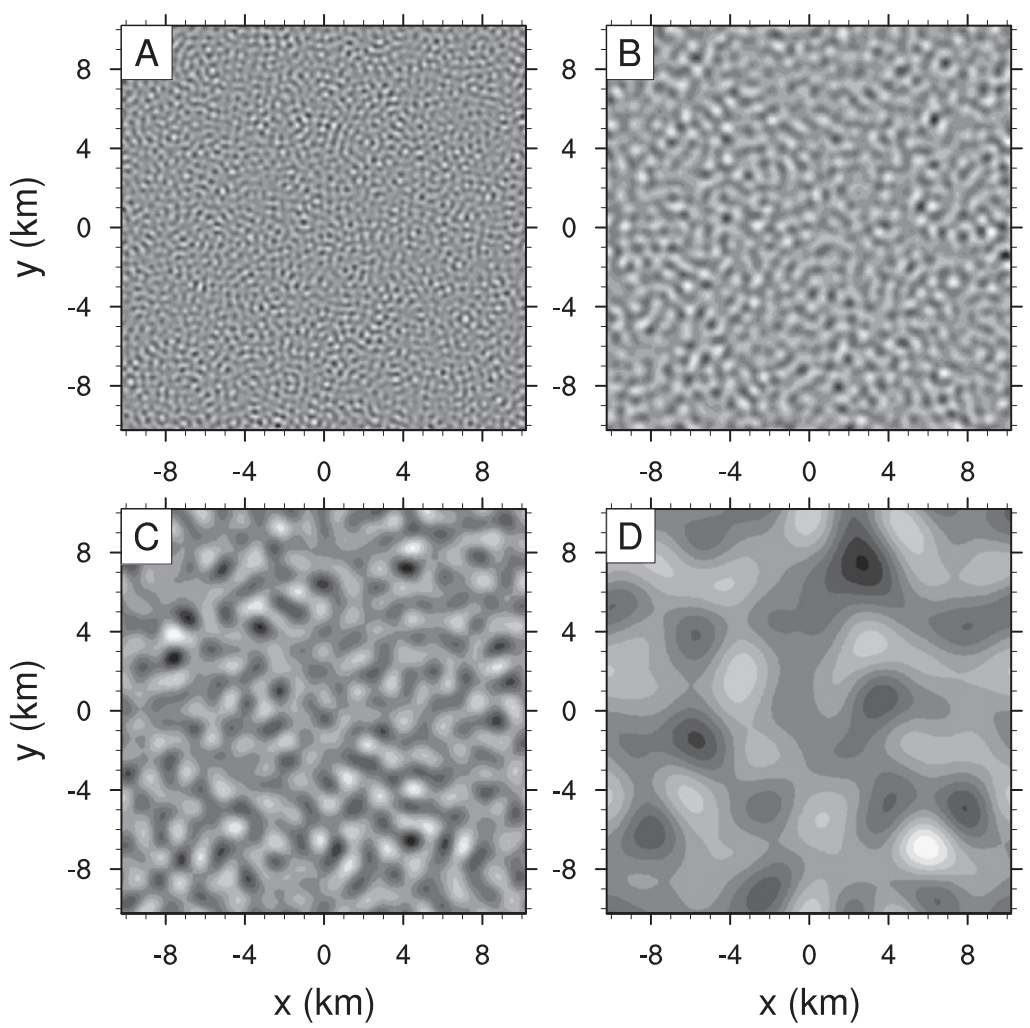

$$
\frac{\partial u_{i}}{\partial x_{j}}(l, m, n) \approx \delta_{j} u_{i}+\mathcal{O}\left(\Delta x_{j}^{2}\right) .
$$

Here $D_{i j}$ is the zero-trace resolved-scale rate of strain tensor:

$$
D_{i j}=\frac{1}{2}\left(\frac{\partial u_{i}}{\partial x_{j}}+\frac{\partial u_{j}}{\partial x_{i}}\right)-\frac{1}{3} \delta_{i j} \frac{\partial u_{k}}{\partial x_{k}} .
$$

\section{APPENDIX B}

\section{Scale of Initial Perturbation and Boundary Layer Variability}

A large part of the observed differences between the present cases involves changes in the character of convection. Although evidence points to sensitivities in the modeling of turbulent transport, the role of initial conditions is briefly discussed in this section. For nonprecipitating cases, the turbulent flow field statistics are expected to decorrelate from the initial condition after a few hours; however, since the onset of precipitation can change the convection structure, an initial condition that causes significant precipitation can have a perpetual effect on the flow.

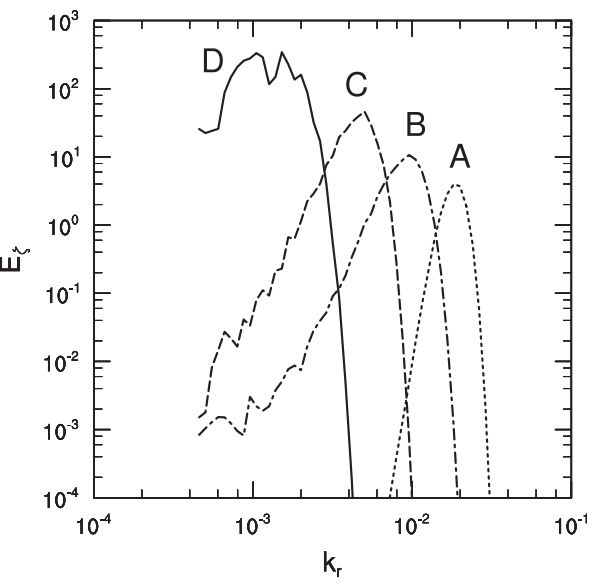

FIG. B1. (left) Initial perturbation fields and (right) their corresponding radial spectra.
In LES of shallow convection a random initial humidity and temperature perturbation is usually added to the lowermost levels of the domain. This perturbation is often discontinuous with a flat spectrum as grid cells are filled with independent random numbers drawn from a uniform distribution. Here, a continuous isotropic random perturbation with a characteristic length scale is constructed.

The method follows that of Cook and Dimotakis (2001). An initially discontinuous random two-dimensional field is filtered with a discrete Gaussian filter to become doubly periodic. Then it is Fourier transformed, Gaussianfiltered, and transformed back to physical space. The Gaussian filter in wavenumber space sets the desired length scale characteristics. Four characteristic length scales are used as shown in Fig. B1 along with their corresponding spectra. In all cases, the perturbation field $\zeta(x, y)$ has zero mean, $\langle\zeta(x, y)\rangle=0$, and the same discrete energy, $\left\langle\zeta(x, y)^{2}\right\rangle=0.0625$.

The perturbation of $q_{t}$ and $\theta_{l}$ in the LES has the form

$$
q_{t}^{\prime}(x, y, z)=0.05\left\langle q_{t}(x, y, 0)\right\rangle \zeta(x, y) \exp \left(\alpha z^{2}\right)
$$

The constant $\alpha$ is chosen such that the perturbation amplitude becomes virtually zero at $z=500 \mathrm{~m}$. For each run different perturbation fields are used for $q_{t}^{\prime}$ and $\theta_{l}^{\prime}$. 

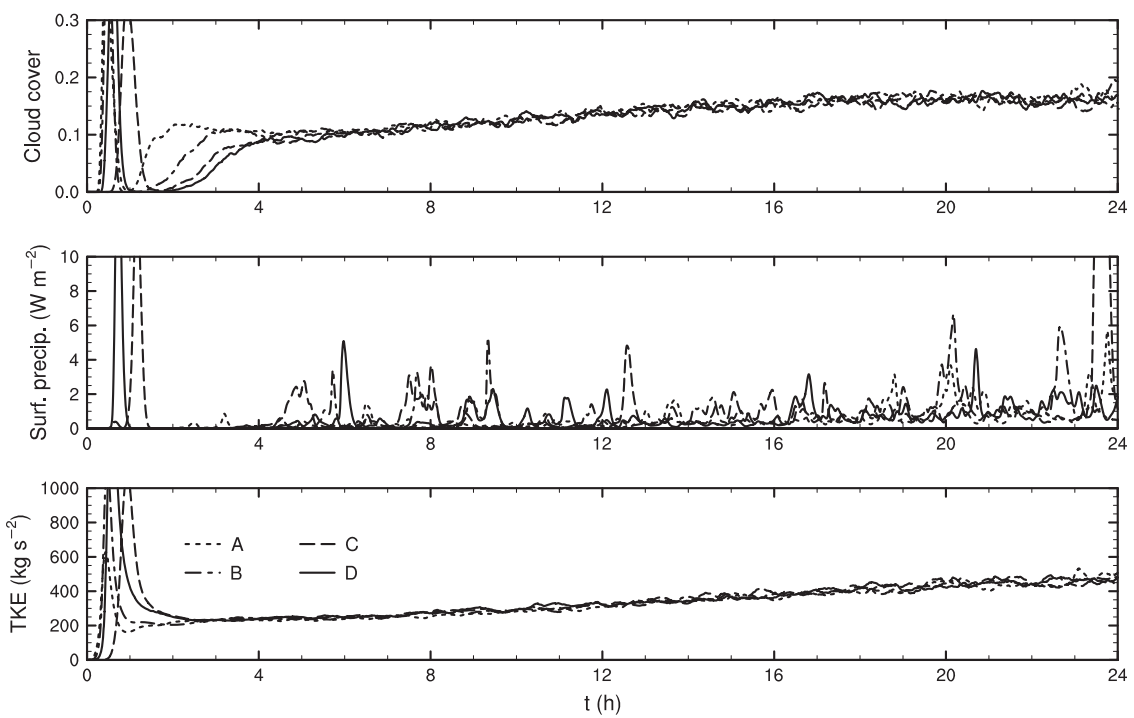

FIG. B2. Time evolution of cloud cover, surface precipitation rate, and vertically integrated TKE for runs initialized with perturbations of varying scales. Line labels are as in Fig. B1.

Four runs corresponding to case SMC-C-40-M were carried out, each initialized with a different perturbation scale. The results of Fig. B2 show no significant effect in late-time statistics of cloud cover, surface precipitation rate, and vertically integrated TKE.

However, there are a few additional conclusions that can be drawn from Fig. B2. As expected, the initial evolution of the boundary layer is strongly dependent on the perturbation length scale. Cloud cover and precipitation rates show that the effect of initial condition persists until $6 \mathrm{~h}$ after initialization. After $6 \mathrm{~h}$ the boundary layer is in a pseudosteady state. Moreover, the time traces show a measure of the variability for these statistics. For the current runs cloud cover is "uncertain" within about $4 \%$. Precipitation is very variable and intermittent. Larger domains are expected to reduce the observed variability in first-order statistics; however, the domain used in the present simulations, $L=20.48 \mathrm{~km}$, is already considered large compared to previous shallow convection studies.

\section{REFERENCES}

Abel, S. J., and B. J. Shipway, 2007: A comparison of cloudresolving model simulations of trade wind cumulus with aircraft observations taken during RICO. Quart. J. Roy. Meteor. Soc., 133, 781-794.

Antonopoulos-Domis, M., 1981: Large-eddy simulation of a passive scalar in isotropic turbulence. J. Fluid Mech., 104, 55-79.

Arakawa, A., 2004: The cumulus parameterization problem: Past, present, and future. J. Climate, 17, 2493-2525.
Bjerknes, J., 1938: Saturated-adiabatic ascent of air through dryadiabatically descending environment. Quart. J. Roy. Meteor. Soc., 64, 325-330.

Bony, S., J.-L. Dufresne, H. Le Treut, J.-J. Morcrette, and C. Senior, 2004: On dynamic and thermodynamic components of cloud changes. Climate Dyn., 22, 71-86.

Brown, A. R., 1999: The sensitivity of large-eddy simulations of shallow cumulus convection to resolution and subgrid model. Quart. J. Roy. Meteor. Soc., 125, 469-482.

Bryan, G. H., J. C. Wyngaard, and J. M. Fritsch, 2003: Resolution requirements for the simulation of deep moist convection. Mon. Wea. Rev., 131, 2394-2416.

Cabot, W., and P. Moin, 1999: Approximate wall boundary conditions in the large-eddy simulation of high Reynolds number flow. Flow Turbul. Combus., 63, 269-291.

Cook, A. W., and P. E. Dimotakis, 2001: Transition stages of Rayleigh-Taylor instability between miscible fluids. J. Fluid Mech., 443, 69-99.

Cuijpers, J. W. M., and P. G. Duynkerke, 1993: Large-eddy simulation of trade wind cumulus clouds. J. Atmos. Sci., 50, 3894-3908.

Ghosal, S., 1996: An analysis of numerical errors in large-eddy simulations of turbulence. J. Comput. Phys., 125, 187-206.

_ 1999: Mathematical and physical constraints on large-eddy simulation of turbulence. AIAA J., 37, 425-433.

Grabowski, W. W., and P. K. Smolarkiewicz, 1990: Monotone finitedifference approximations to the advection-condensation problem. Mon. Wea. Rev., 118, 2082-2097.

Harlow, F. H., and J. E. Welch, 1965: Numerical calculation of time-dependent viscous incompressible flow of fluid with free surface. Phys. Fluids, 8, 2182-2189.

Heus, T., and H. J. J. Jonker, 2008: Subsiding shells around shallow cumulus clouds. J. Atmos. Sci., 65, 1003-1018.

Kamakoti, R., and C. Pantano, 2009: High-order narrow stencil finite-difference approximations of second-order derivatives involving variable coefficients. SIAM J. Sci. Comput., 31, $4222-4243$. 
Lilly, D. K., 1962: On the numerical simulation of buoyant convection. Tellus, 14, 148-172.

Malkus, J. S., 1954: Some results of a trade-cumulus cloud investigation. J. Meteor., 11, 220-237.

Mason, P. J., 1994: Large-eddy simulation: A critical review of the technique. Quart. J. Roy. Meteor. Soc., 120, 1-26.

- , and N. S. Callen, 1986: On the magnitude of the subgrid-scale eddy coefficient in large-eddy simulations of turbulent channel flow. J. Fluid Mech., 162, 439-462.

— eddy simulations of boundary layers. J. Fluid Mech., 242, 51-78.

Matheou, G., A. M. Bonanos, C. Pantano, and P. E. Dimotakis, 2010: Large-eddy simulation of mixing in a recirculating shear flow. J. Fluid Mech., 646, 375-414.

Morinishi, Y., T. S. Lund, O. V. Vasilyev, and P. Moin, 1998: Fully conservative higher order finite difference schemes for incompressible flow. J. Comput. Phys., 143, 90-124.

Neggers, R. A. J., M. Köhler, and A. C. M. Beljaars, 2009: A dual mass flux framework for boundary layer convection. Part I: Transport. J. Atmos. Sci., 66, 1465-1487.

Nieuwstadt, F. T. M., P. J. Mason, C.-H. Moeng, and U. Schumann, 1993: Large-eddy simulation of the convective boundary layer: A comparison of four computer codes. Turbulent Shear Flows 8, F. Durst et al., Eds., SpringerVerlag, 344-367.

Nuijens, L., B. Stevens, and A. P. Siebesma, 2009: The environment of precipitating shallow cumulus convection. J. Atmos. Sci., 66, 1962-1979.

Ogura, Y., and N. A. Phillips, 1962: Scale analysis of deep and shallow convection in the atmosphere. J. Atmos. Sci., 19, 173-179.

Pantano, C., R. Deiterding, D. J. Hill, and D. I. Pullin, 2007: A lownumerical dissipation patch-based adaptive mesh refinement method for large-eddy simulation of compressible flows. J. Comput. Phys., 221, 63-87.

Piacsek, S. A., and G. P. Williams, 1970: Conservation properties of convection difference schemes. J. Comput. Phys., 6, 392-405.

Piotrowski, Z. P., P. K. Smolarkiewicz, S. P. Malinowski, and A. A. Wyszogrodzki, 2009: On numerical realizability of thermal convection. J. Comput. Phys., 228, 6268-6290.

Pope, S. B., 2004: Ten questions concerning the large-eddy simulation of turbulent flows. New J. Phys., 6, 35.

Randall, D., M. Khairoutdinov, A. Arakawa, and W. Grabowski, 2003: Breaking the cloud parameterization deadlock. Bull. Amer. Meteor. Soc., 84, 1547-1564.

Rauber, R. M., N. F. Laird, and H. T. Ochs III, 1996: Precipitation efficiency of trade wind clouds over the north central tropical Pacific Ocean. J. Geophys. Res., 101 (D21), $26247-26253$.

— , and Coauthors, 2007: Rain in shallow cumulus over the ocean: The RICO campaign. Bull. Amer. Meteor. Soc., 88, 1912-1928.

Savic-Jovcic, V., and B. Stevens, 2008: The structure and mesoscale organization of precipitating stratocumulus. J. Atmos. Sci., 65, 1587-1605.

Schmidt, H., and U. Schumann, 1989: Coherent structure of the convective boundary layer derived from large-eddy simulations. J. Fluid Mech., 200, 511-562.

Seifert, A., and K. D. Beheng, 2001: A double-moment parameterization for simulating autoconversion, accretion and selfcollection. Atmos. Res., 59-60, 265-281.
Short, D. A., and K. Nakamura, 2000: TRMM radar observations of shallow precipitation over the tropical oceans. J. Climate, 13, 4107-4124.

Siebesma, A. P., 1998: Shallow cumulus convection. Buoyant Convection in Geophysical Flows, E. J. Plate et al., Eds., Kluwer Academic, 441-486.

— , and A. A. M. Holtslag, 1996: Model impacts of entrainment and detrainment rates in shallow cumulus convection. J. Atmos. Sci., 53, 2354-2364.

_ , and H. J. J. Jonker, 2000: Anomalous scaling of cumulus cloud boundaries. Phys. Rev. Lett., 85, 214-217.

—_, and Coauthors, 2003: A large eddy simulation intercomparison study of shallow cumulus convection. J. Atmos. Sci., 60, 12011219.

— , P. M. M. Soares, and J. Teixeira, 2007: A combined eddydiffusivity mass-flux approach for the convective boundary layer. J. Atmos. Sci., 64, 1230-1248.

Skamarock, W. C., 2004: Evaluating mesoscale NWP models using kinetic energy spectra. Mon. Wea. Rev., 132, 30193032 .

— and M. L. Weisman, 2009: The impact of positive-definite moisture transport on NWP precipitation forecasts. Mon. Wea. Rev., 137, 488-494.

Smagorinsky, J., 1963: General circulation experiments with the primitive equations. I. The basic experiment. Mon. Wea. Rev., 91, 99-164.

Snodgrass, E. R., L. Di Girolamo, and R. M. Rauber, 2009: Precipitation characteristics of trade wind clouds during RICO derived from radar, satellite, and aircraft measurements. J. Appl. Meteor., 48, 464-483.

Soares, P. M. M., P. M. A. Miranda, A. P. Siebesma, and J. Teixeira, 2004: An eddy-diffusivity/mass-flux parametrization for dry and shallow cumulus convection. Quart. J. Roy. Meteor. Soc., 130, 3365-3383.

Sommeria, G., 1976: Three-dimensional simulation of turbulent processes in an undisturbed trade wind boundary layer. J. Atmos. Sci., 33, 216-241.

— in models of nonprecipitating clouds. J. Atmos. Sci., 34, 344-355.

Spalart, P. R., R. D. Moser, and M. M. Rogers, 1991: Spectral methods for the Navier-Stokes equations with one infinite and two periodic directions. J. Comput. Phys., 96, 297-324.

Stevens, B., 2005: Atmospheric moist convection. Annu. Rev. Earth Planet. Sci., 33, 605-643.

__, and A. Seifert, 2008: Understanding macrophysical outcomes of microphysical choices in simulations of shallow cumulus convection. J. Meteor. Soc. Japan, 86A, 143-162.

— C. H. Moeng, and P. P. Sullivan, 1999: Large-eddy simulations of radiatively driven convection: Sensitivities to the representation of small scales. J. Atmos. Sci., 56, 39633984.

—_ and Coauthors, 2001: Simulations of trade wind cumuli under a strong inversion. J. Atmos. Sci., 58, 1870-1891.

— , and Coauthors, 2005: Evaluation of large-eddy simulations via observations of nocturnal marine stratocumulus. Mon. Wea. Rev., 133, 1443-1462.

Stevens, D. E., A. S. Ackerman, and C. S. Bretherton, 2002: Effects of domain size and numerical resolution on the simulation of shallow cumulus convection. J. Atmos. Sci., 59, 3285-3301. 
Stommel, H., 1947: Entrainment of air into a cumulus cloud. J. Meteor., 4, 91-94.

Stull, R. B., 1988: An Introduction to Boundary Layer Meteorology. Kluwer Academic, 666 pp.

Teixeira, J., and Coauthors, 2008: Parameterization of the atmospheric boundary layer: A view from just above the inversion. Bull. Amer. Meteor. Soc., 89, 453-458.

Tiedtke, M., 1989: A comprehensive mass flux scheme for cumulus parameterization in large-scale models. Mon. Wea. Rev., 117, $1779-1800$

Toro, E. F., 1999: Riemann Solvers and Numerical Methods for Fluid Dynamics: A Practical Introduction. 2nd ed. Springer, $624 \mathrm{pp}$.
Vallis, G. K., 2006: Atmospheric and Oceanic Fluid Dynamics: Fundamentals and Large-Scale Circulation. Cambridge University Press, 745 pp.

VanZanten, M. C., and Coauthors, 2011: Controls on precipitation and cloudiness in simulations of trade-wind cumulus as observed during RICO. J. Adv. Model. Earth Syst., 3, M06001, doi:10.1029/2011MS000056.

Viré, A., and B. Knaepen, 2009: On discretization errors and subgrid scale model implementations in large eddy simulations. J. Comput. Phys., 228, 8203-8213.

Xue, H., G. Feingold, and B. Stevens, 2008: Aerosol effects on clouds, precipitation, and the organization of shallow cumulus convection. J. Atmos. Sci., 65, 392-406. 